\title{
Data Processing Package for Cyclic Integrated Reversible Bending Fatigue Testing
}

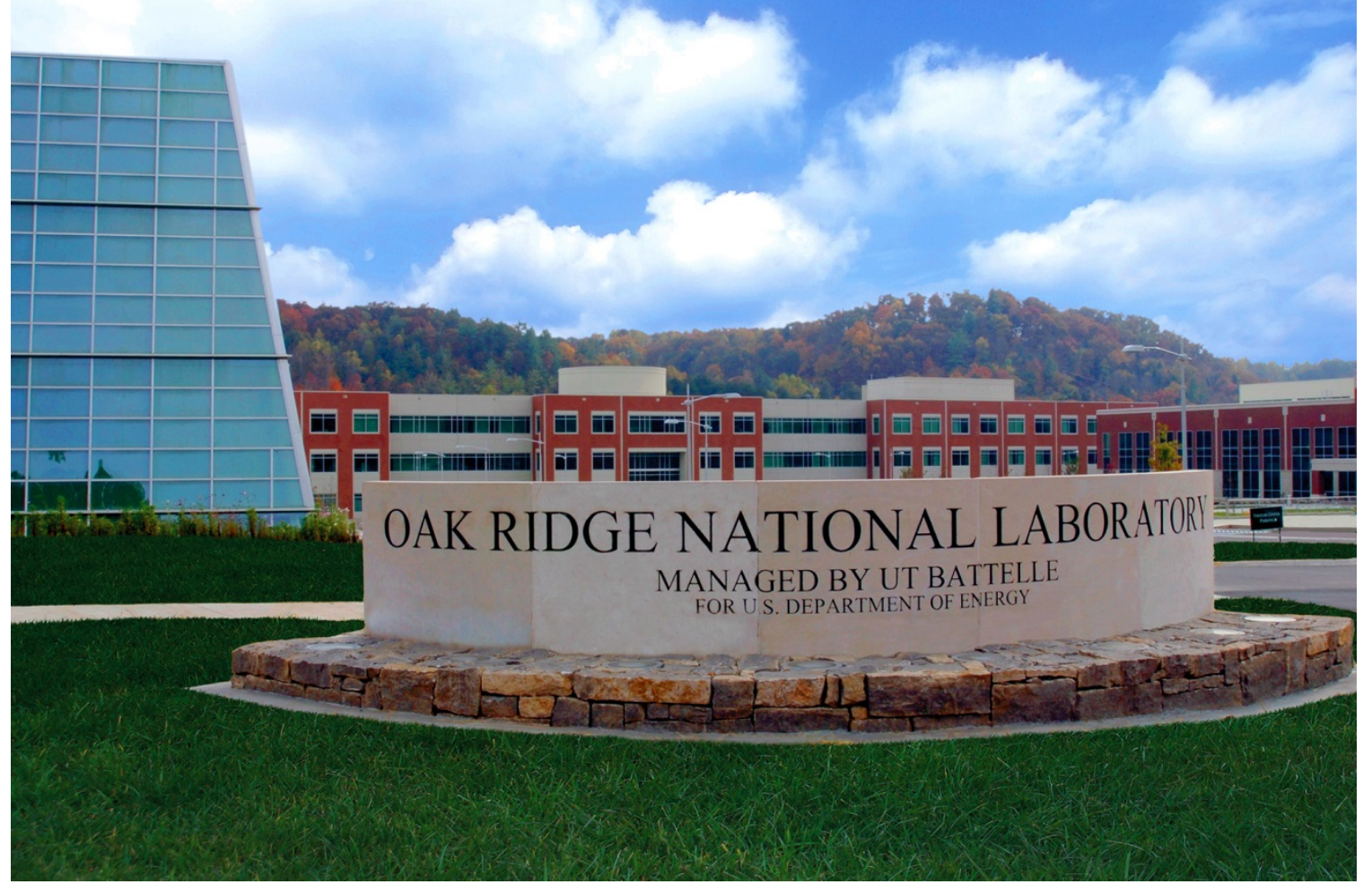

Hong Wang

Jy-An John Wang

July 2021 


\title{
DOCUMENT AVAILABILITY
}

Reports produced after January 1, 1996, are generally available free via US Department of Energy (DOE) SciTech Connect.

Website www.osti.gov

Reports produced before January 1, 1996, may be purchased by members of the public from the following source:

\author{
National Technical Information Service \\ 5285 Port Royal Road \\ Springfield, VA 22161 \\ Telephone 703-605-6000 (1-800-553-6847) \\ TDD 703-487-4639 \\ Fax 703-605-6900 \\ E-mail info@ntis.gov \\ Website http://classic.ntis.gov/
}

Reports are available to DOE employees, DOE contractors, Energy Technology Data Exchange representatives, and International Nuclear Information System representatives from the following source:

Office of Scientific and Technical Information

PO Box 62

Oak Ridge, TN 37831

Telephone 865-576-8401

Fax 865-576-5728

E-mail reports@osti.gov

Website http://www.osti.gov/

This report was prepared as an account of work sponsored by an agency of the United States Government. Neither the United States Government nor any agency thereof, nor any of their employees, makes any warranty, express or implied, or assumes any legal liability or responsibility for the accuracy, completeness, or usefulness of any information, apparatus, product, or process disclosed, or represents that its use would not infringe privately owned rights. Reference herein to any specific commercial product, process, or service by trade name, trademark, manufacturer, or otherwise, does not necessarily constitute or imply its endorsement, recommendation, or favoring by the United States Government or any agency thereof. The views and opinions of authors expressed herein do not necessarily state or reflect those of the United States Government or any agency thereof. 
Materials Science and Technology Division

\title{
DATA PROCESSING PACKAGE FOR CYCLIC INTEGRATED REVERSIBLE BENDING FATIGUE TESTING
}

\author{
Hong Wang, Jy-An John Wang
}

\section{Program Manager}

Bruce B. Bevard

Nuclear Energy and Fuel Cycle Division

July 2021

Prepared by OAK RIDGE NATIONAL LABORATORY

Oak Ridge, TN 37831-6283

managed by

UT-BATTELLE, LLC

for the

US DEPARTMENT OF ENERGY

under contract DE-AC05-00OR22725 



\section{CONTENTS}

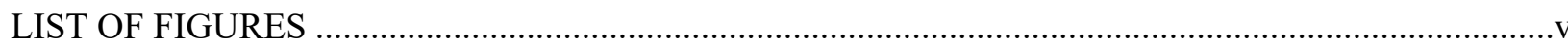

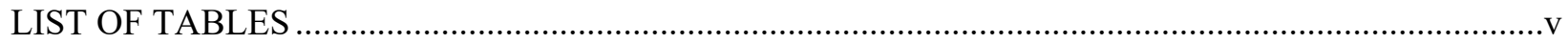

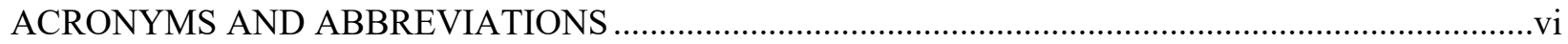

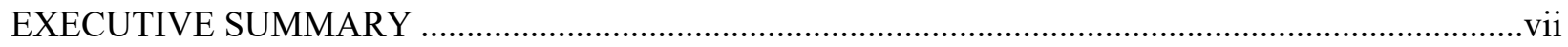

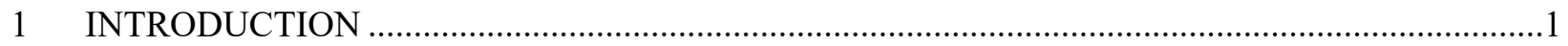

1.1 FEDERAL REQUIREMENTS FOR TRANSPORTATION OF SPENT NUCLEAR

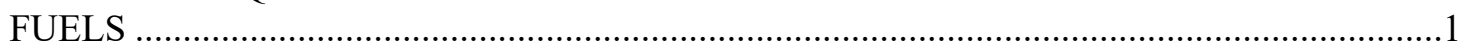

1.2 BASIC CONSIDERATIONS ON TESTING SYSTEM AND TESTING DATA.....................

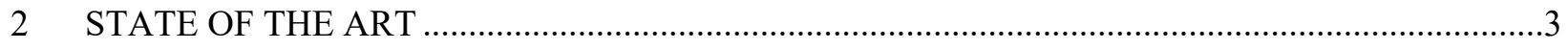

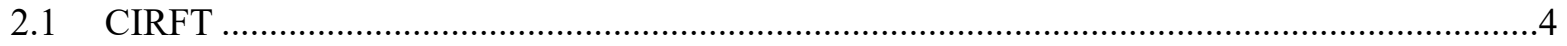

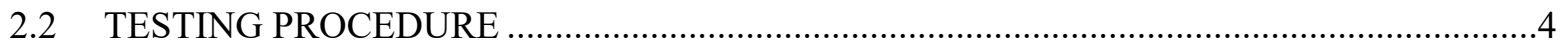

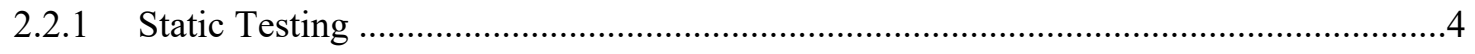

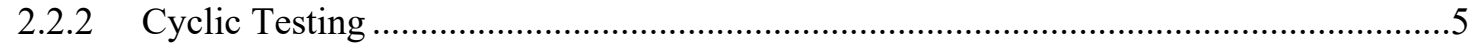

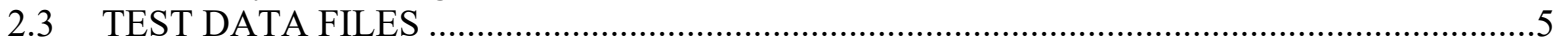

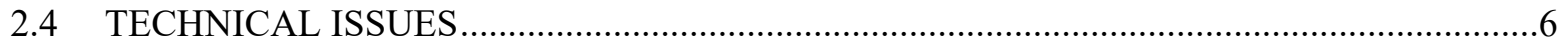

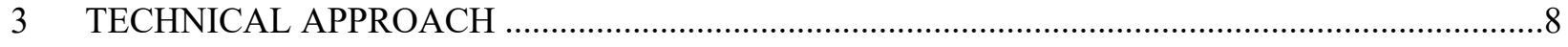

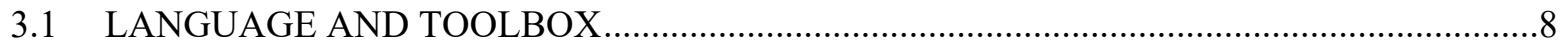

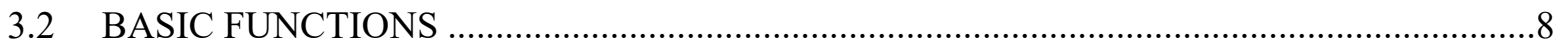

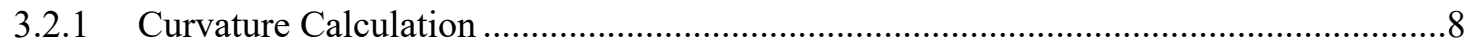

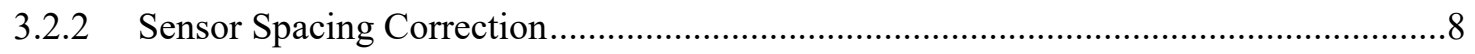

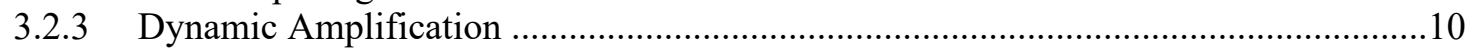

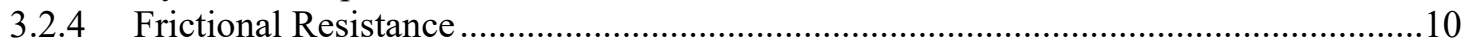

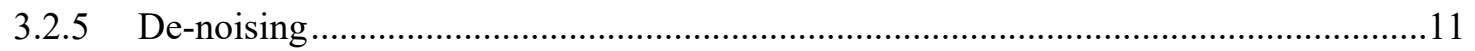

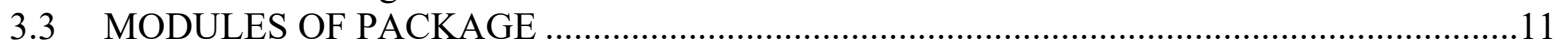

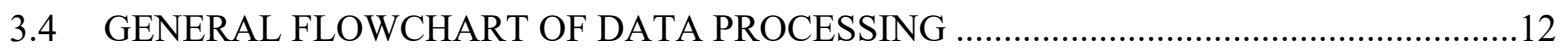

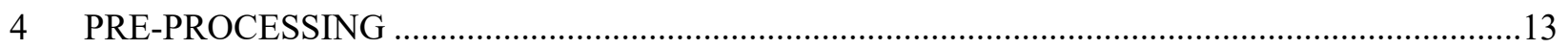

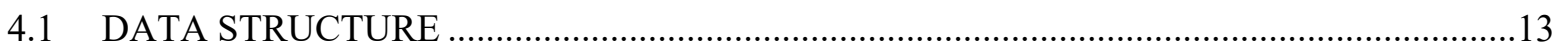

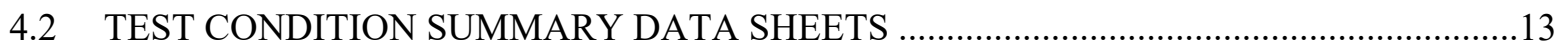

4.2.1 Test Summary for Individual Specimens ............................................................... 13

4.2.2 Test and Specimen Status Summaries for Group ................................................13

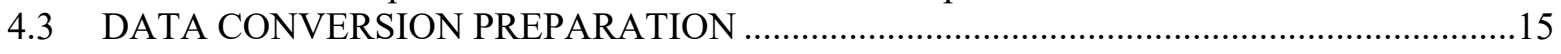

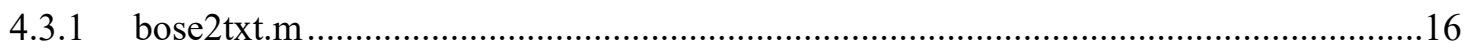

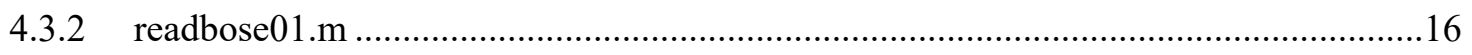

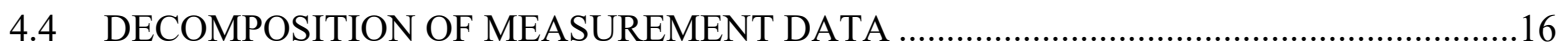

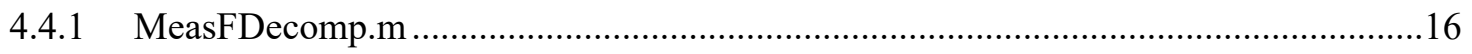

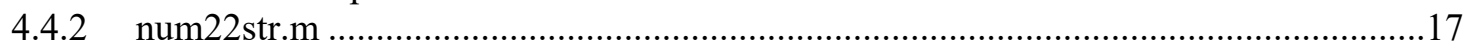

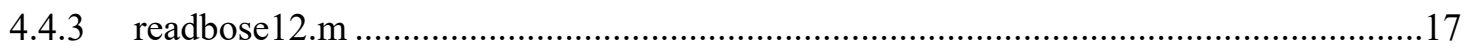

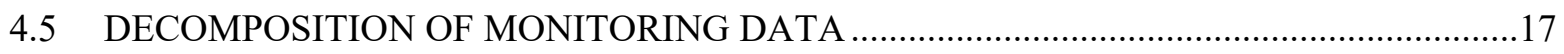

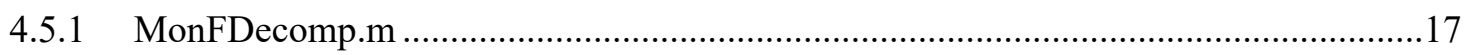

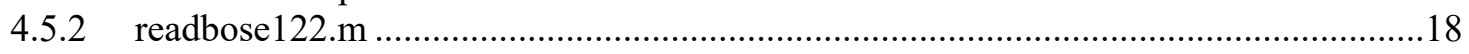

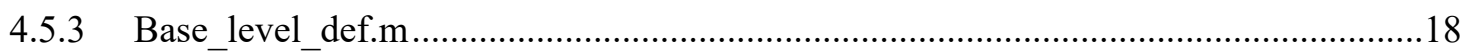

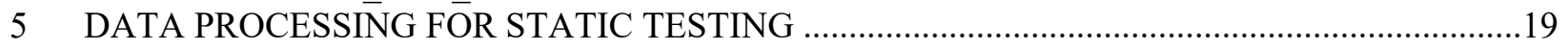

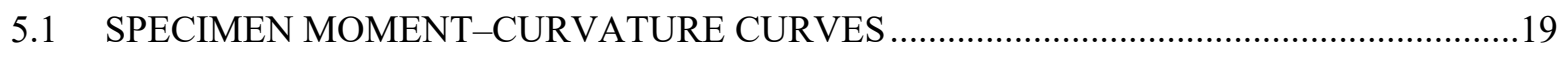

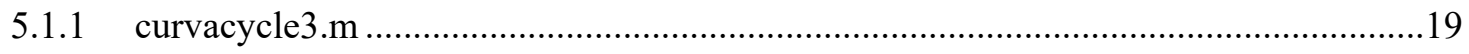

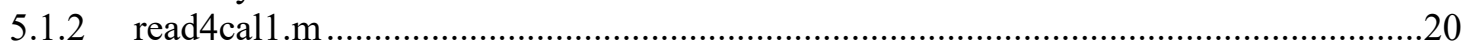

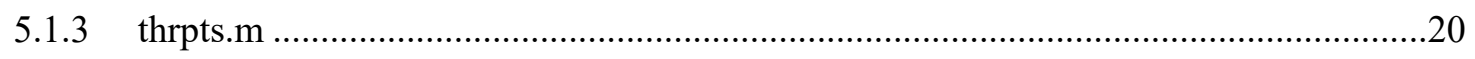

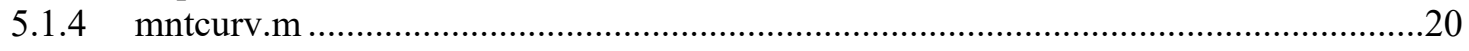

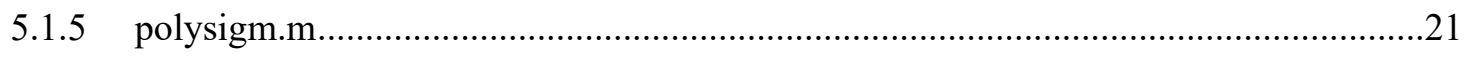




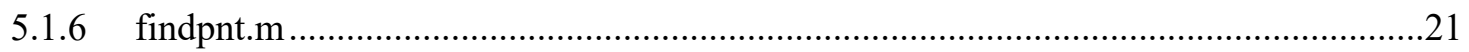

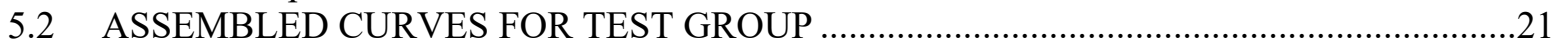

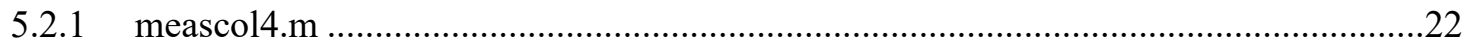

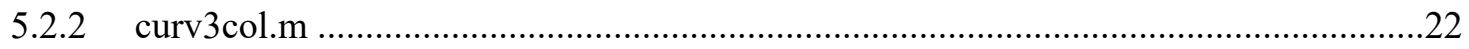

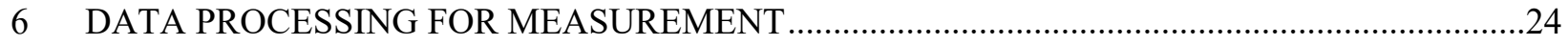

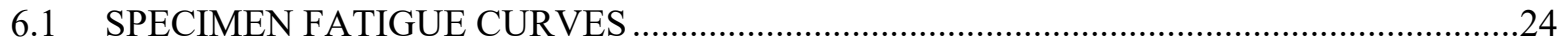

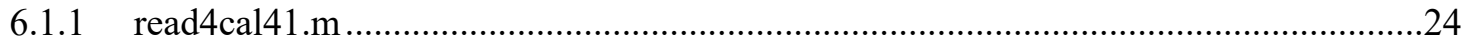

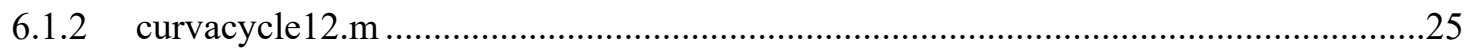

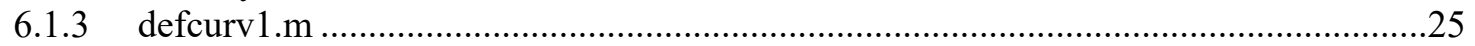

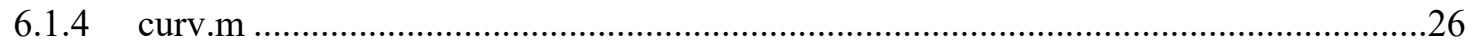

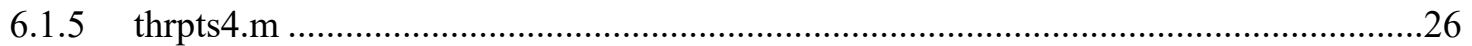

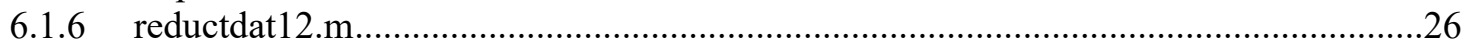

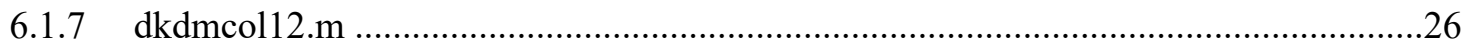

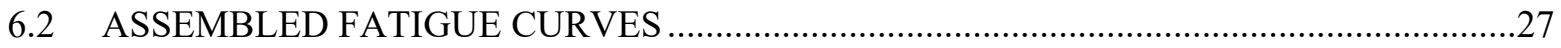

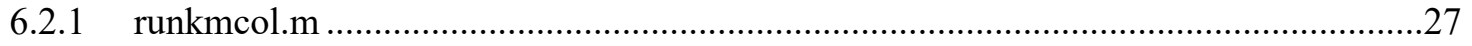

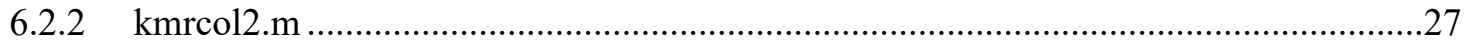

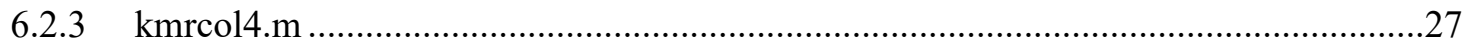

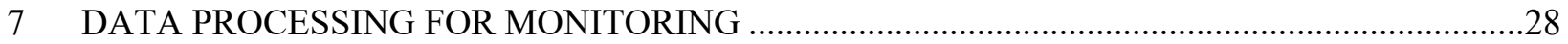

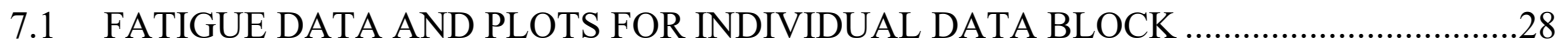

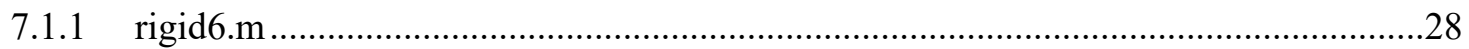

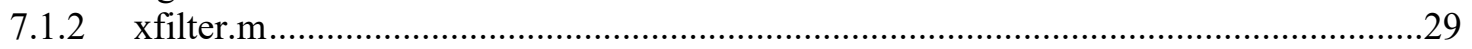

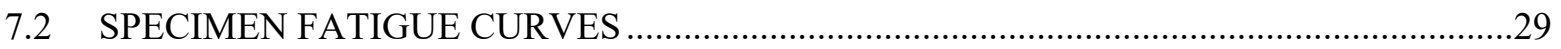

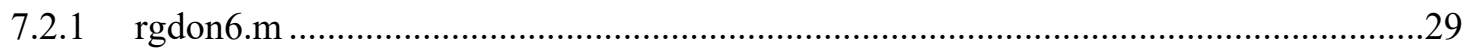

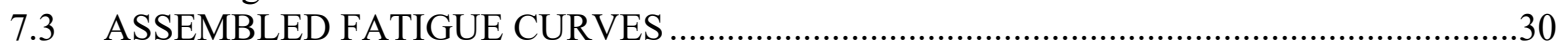

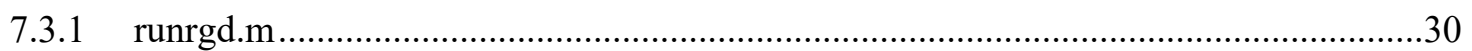

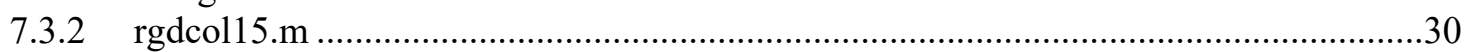

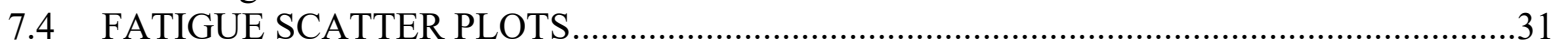

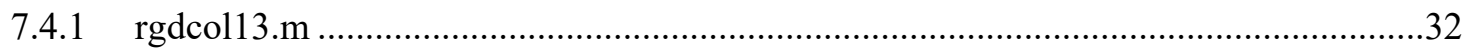

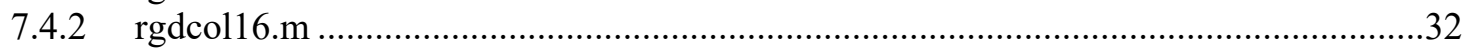

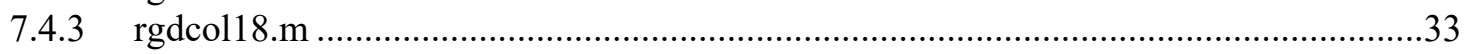

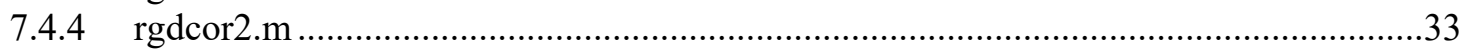

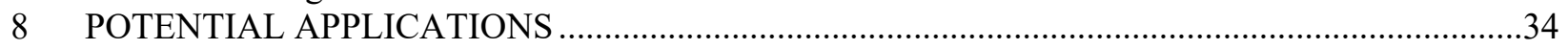

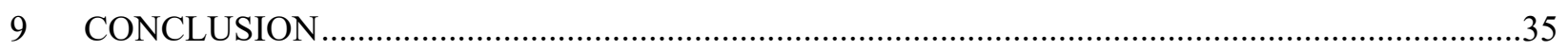

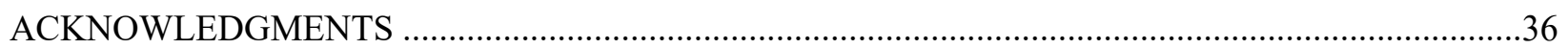

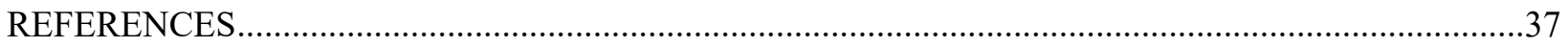




\section{LIST OF FIGURES}

Figure 1. Flowchart illustrating a typical CIRFT process.....................................................................5

Figure 2. Schematic of scans/data blocks in a typical cyclic test: (a) test sessions, (a) scans of monitoring, (c) scans of measurement, (d) and (e) individual scans/ data blocks. .........................6

Figure 3. Diagram of three deflections used in curvature calculation where sensor spacing is $h$...............8

Figure 4. Diagram of curvature corrections when linear motors are in (a) pull, (b) neutral position, and (c) push

Figure 5. A schematic showing the spacing correction applied to correct the curvature and adjust the curvature profile.

Figure 6. Schematic showing the load profiles of (a) a static test, (b) an empty test, and (c) a test with the effect of frictional resistance removed.

Figure 7. Schematic to illustrate the procedure used to remove noise in curvature data: (a) raw signal, (b) linear spectrum, (c) spectrum after filtering, (d) signal reconstructed, and (e) residuals.

Figure 8. Modules for data processing.

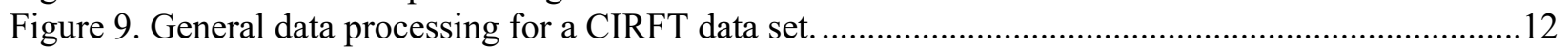

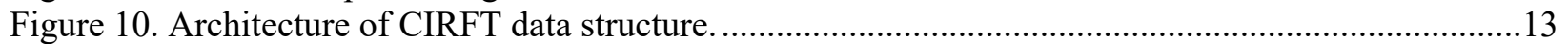

Figure 11. Flowchart of data processing for static test. .........................................................................19

Figure 12. A schematic showing the quantities used in characterization. ............................................21

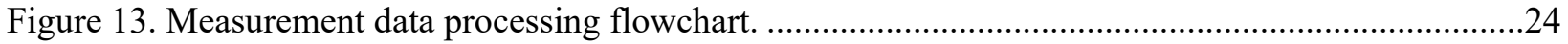

Figure 14. Quantities are introduced above to characterize the bending mechanical properties of

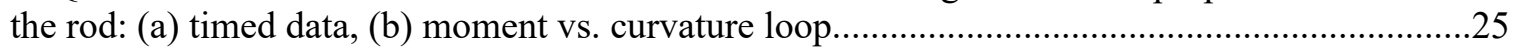

Figure 15. Flowchart of monitoring data processing and functions. ...................................................28

Figure 16. Diagram showing koffset adjustment related to the measurement interruption or

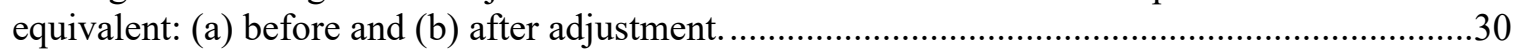

\section{LIST OF TABLES}

Table 1. Example of data sheet summarizing tests on a specimen. ......................................................13

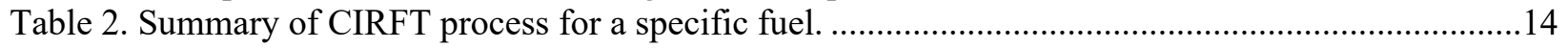

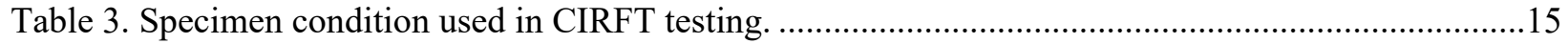

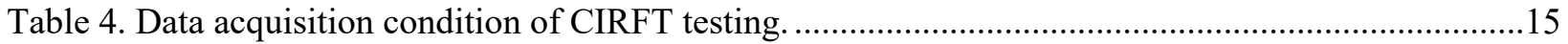




\section{ACRONYMS AND ABBREVIATIONS}

$\begin{array}{ll}\text { BU } & \text { Burn- up } \\ \text { CIRFT } & \text { Cyclic Integrated Reversible-Bending Fatigue Tester/ Testing } \\ \text { CLD } & \text { cladding type } \\ \text { CTK } & \text { cladding wall thickness } \\ \text { DDR } & \text { Data directory } \\ \text { DM } & \text { Moment range in cycling } \\ \text { DP } & \text { Load range in cycling } \\ \text { FFT } & \text { Fast Fourier Transform } \\ \text { ID } & \text { Inside diameter, identity } \\ \text { LE } & \text { Low elevation of rod segment } \\ \text { LVDT } & \text { Linear variable differential transducer } \\ \text { OD } & \text { Outside diameter } \\ \text { ORNL } & \text { Oak Ridge National Laboratory } \\ \text { PD } & \text { Pellet diameter } \\ \text { PL } & \text { Pellet length } \\ \text { RID } & \text { Rod segment identity } \\ \text { SID } & \text { Specimen identity } \\ \text { SNF } & \text { Spent nuclear fuel } \\ \text { SS } & \text { Sensor spacing } \\ \text { STT } & \text { Statue of specimen } \\ \text { TD } & \text { Timed data } \\ \text { UFDC } & \text { Used Fuel Disposition Campaign }\end{array}$




\section{EXECUTIVE SUMMARY}

Oak Ridge National Laboratory (ORNL) recently has developed a test system, called as cyclic integrated reversible bending fatigue tester (CIRFT), to address the technical need in testing and evaluation of vibration integrity of high burn-up (BU) spent nuclear fuel (SNF) rods. A large volume of data is usually generated from periodic measurements and online monitoring in a typical cyclic test. The amount of data can become quite sizable for a group of specimens. Moreover, the CIRFT uses the curvature to measure the deformation of rod specimen that is based on the readings from three linear variable differential transducers (LVDTs). The interaction of the LVDT probes with the rod surface complicates the measurement and presents considerable challenge to subsequent data analysis. The commercial software in mechanical testing field is limited and cannot meet the data processing requirements associated with CIRFT application.

This report describes the main components of a data processing software package dedicated to CIRFT. The package consists of four modules: pre-processing, data processing for static testing, data processing for monitoring, and data processing for measurements. Users have the option to select modules for specified tasks. The package has been developed using MATLAB. Curve Fitting Toolbox is needed to run some of the programs. The report describes the functionality, data flow, input \& output of each function. An example is provided to help users start the program.

CIRFT uses Bose WinTest7 for test control and data acquisition. The Bose output file in a test session is configured with heading and data columns related to the following channels: displacements and loads of two linear motors, and deflections of three LVDTs at three points of the gage section.

The test results obtained from data processing are presented in curves, scatter plots, and tables. For static testing, the output in tables provides bending mechanical properties and characteristic points of momentcurvature relation: flexural rigidities in linear segments of loading and unloading stages, intersection points between characteristic segments of the curve, and equivalent stress and strain quantities.

For cyclic testing, the table output lists control, fatigue life, and responsive/dependent quantities: ranges (peak-to-peak values) of moment and curvature, flexural rigidity, flexural hysteresis or rigidity phase angle, and ranges of equivalent stresses and strains. In addition, derivatives such as half-gage length and sensor spacing correction are included. The output also provides standard deviations of the reported quantities when they are applicable or available.

The data processing package can serve as a fundamental characterization tool in mechanical study of materials. The package is intended primarily for data processing for the CIRFT process and can also be used in applications for which similar testing requirements exist. The package is a critical element for CIRFT to be developed as an important nuclear testing facility and for many end users in the field of storage, handling, and transportation of used fuels. 


\section{INTRODUCTION}

\subsection{FEDERAL REQUIREMENTS FOR TRANSPORTATION OF SPENT NUCLEAR FUELS}

Transportation packages of spent nuclear fuel (SNF) must meet safety requirements under normal and accident conditions, as specified by federal regulations. For normal conditions of transport, it is required that the licensee ensures that there will be no loss or dispersal of spent fuel, no significant increase in external surface radiation levels, and no substantial reduction in the effectiveness of the spent fuel package. These requirements include vibration loads related to transport. ${ }^{1}$

For SNF transportation, the fuel assemblies are loaded in a transportation cask or in a dual-purpose canister with a transportation overpack. The transportation cask is then loaded onto a truck or railcar and is usually transported in a horizontal position. The interaction between the vehicle and the ground creates vibration, which in turn is transmitted into the transportation cask internal structure (baskets) and fuel assembly spacer grids.

Spent fuel rod vibration during normal transport is important because of the sustained loads involved; it is particularly important for high-burnup (high-BU) fuel ( $>45 \mathrm{GWd} / \mathrm{MTU})$. As the BU of the fuel increases, a number of changes occur that may affect the performance of the fuel and cladding in storage and transportation. These changes include increased cladding corrosion layer thickness, increased cladding hydrogen content, increased cladding creep strains, increased fission gas release, and formation of a highBU structure at the surfaces of the fuel pellets. For example, the presence of hydrides can facilitate cracking if the hydrides are aligned radially, perpendicular to the tensile stress field. ${ }^{2}$

Therefore, the evaluation of spent fuel rod integrity under vibration loading is an important part of the safety assessments needed for the extended storage and transportation of high-BU SNF.

\subsection{BASIC CONSIDERATIONS ON TESTING SYSTEM AND TESTING DATA}

This research focuses on single fuel rods. Specifically, the project that has resulted in the data processing package discussed in this report investigates an experimental technology to study rod segments using a mechanical testing machine in a hot cell. There are many technical challenges involved that need to be addressed carefully for this research and development.

- Lack of accessibility: The testing system is not directly accessible once placed in a hot cell.

Implementation of any experimental technology is expected to be simple and easy for a manipulator to operate in a hot cell.

- Complicated failure modes: The inertia-induced loading to the rods during the transportation of SNF is dominated by bending vibrations. The testing system developed shall capture such loading modes.

- Unique high-BU SNF condition: The SNF rod generally features internal and surface structural damage. Specimens with reduced gage sections are suggested. Therefore, for a straight SNF rod segment, endcaps are needed to ensure failure occurs in the gage section. Thus, a measure is also needed to protect the capped specimen from contact damage.

- High-radioactivity environment: All critical components of the testing system are expected to be durable in such a hostile environment.

Testing data must offer the basis for subsequently characterizing and evaluating the mechanical properties of rods under equivalent loading conditions. For the type of bending fatigue under investigation, the 
fatigue life, or number of cycles to failure, is generally as obtained as in conventional fatigue testing. The fatigue responses of a rod in cycling also provide the critical information to understand the mechanisms that result in final failure. The responses include bending moment and deformation of rods under a loadcontrol mode. The fatigue responses should provide the potential of predicting the fatigue behavior of a rod and to justify the lifetime data for the rod and the performance of testing system.

A test protocol must therefore be designed carefully and implemented to ensure that the objectives are achieved. For bending fatigue, the protocol covers both static and cyclic tests. A group of specimens are generally divided into static and cyclic testing subgroups. The static testing provides the overall response of a rod under large deformation and serves as input for the design of the subsequent cyclic testing of the rod. The measurement and continuous monitoring are expected to be enabled to capture the fatigue responses of the rod. 


\section{STATE OF THE ART}

The dynamic loads to SNF rods during transportation are characteristic of bending vibration. The vibration signals in a specified transport environment can be analyzed and frequency components can be identified. Hence, the vibration integrity of SNF rods in transportation can be studied through testing and evaluation of the fatigue performance of SNF rods at defined frequencies. Bending fatigue testing has been practiced for some time..$^{3-6}$ According to the beam boundary condition, the testing can be classified as cantilever beam flexure, ${ }^{7}$ three-point bending, four-point bending, ${ }^{8-11}$ and pure bending. ${ }^{12}$ Bending fatigue tests also include variants that have been developed in response to environmental factors, particularly temperature. ${ }^{13}$

Four-point bending fatigue has been proved to be a mature experimental technique for testing materials and components that have limited deformation before failure. The technique is mostly used in fatigue tests without bending reversal. Four-point/three-point bending and cantilever bending all experience the inherent drawbacks related to transverse shear, which has a significant impact on materials that are sensitive to shear.

Pure bending test methods are preferred for materials whose failure is sensitive to shear. ${ }^{14-16}$ A pure bending fatigue machine for reverse bending was first developed by Forrest and Penfold. ${ }^{17}$ It was modified and used by UK turbine makers in testing high-strain fatigue related to thermal transients in low-alloy steels. ${ }^{18,19}$ The beam specimen was held by two cross-shafts. The cross-shafts were free to rotate, and one of them was also free to move to release axial loading on the specimen. The rotation of cross-shafts was introduced through two crank-arms and links that were driven by an electric motor via gearboxes. Results for CrMoV-type steels under displacement-controlled reverse-bend conditions provided similar endurance data to tests conducted under direct strain-controlled push-pull conditions. ${ }^{19}$ The fatigue machine generally worked at a low frequency (less than $1 \mathrm{~Hz}$ ) that is of interest to creep fatigue study. The system lacked strain control in the specified control mode. Another pure bending approach is derived from the four-point bending principle. Similar to the flexure-based device, ${ }^{10}$ the implementation places the specimen under test in an inner span and reinforces the specimen-retaining part (the end parts of the outer span) with rigid sleeves. It is the rigid sleeves that convert the external force couples into bending moments applied to the specimen. ${ }^{12}$ Compliant layers are utilized as transits between the rigid sleeves and the specimen to control potential contact damage. Supports are provided by a position system equipped with rollers, and loading points are constructed by the use of pivots on slide connections attached to a swing beam. The configuration of the setup ensures the release of axial stresses and can handle the large deformation fatigue experienced by ductile materials during fatigue. However, cyclic bending can be conducted only in non-reversal or unidirectional mode.

The following are the main observations for the status of bending fatigue testing:

- Various supports have been developed for bending fatigue testing, including flexures, ${ }^{10}$ rotary joints, ${ }^{11}$ slide connections, ${ }^{12}$ and motorized clamps. ${ }^{20}$ They are neither demonstrated nor enabled in reverse mode.

- Pure bending fatigue has been used for high strain and low cycle fatigue in testing metallic alloys and composites. The implementation of the pure bending concept is usually application-based with partial success.

- Environmental chambers and/or high-temperature furnaces are currently incorporated into some critical bending fatigue tests. The setup of specimens and tests is all accomplished manually. 
The existing experimental methods and testing systems cannot meet the requirements of vibration studies of SNFs. To address the technical gap, Wang et al. recently developed a system called a cyclic integrated reversible bending fatigue tester (CIRFT) that is dedicated to testing SNF rods under pure bending conditions in a hot cell. The system is set up on a testing bench and uses a U-frame to convert the linear motion into bending moment applied to the rod specimen under test. The U-frame serves as a mechanism that integrates all the functional elements, unlike conventional bending systems that use a set of discrete components for test setup. The CIRFT approach adopted thus simplifies the operation involved with the specimen setup in a hot cell. Relevant details of the system components, design considerations, and calibration can be found in several publications. ${ }^{21-29}$ Details of the use of the system in testing a variety of SNF rods and relevant testing data can be found in additional references. ${ }^{30-47}$

\subsection{CIRFT}

The CIRFT consists of dual Bose linear motors ${ }^{48}$ that are set up on a testing bench with a U-shaped loading frame. The dual linear motors are aligned longitudinally and driven lineally toward or away from each other. The U-frame converts the linear motion into bending of the specimen. The grips, installed within dual rigid arms that can release any axial force, apply pure bending to the specimen within the gage section. The system measures the deformation of rod by using three linear variable differential transducers (LVDTs) installed in the gage section. In this way, the curvature can be determined. This is different from the conventional bending test, in which the deflection is used for characterization. In CIRFT, a compliant layer is used in endcaps of specimen grips to protect the specimen from any contact damages. The compliant layer, with additional deformation introduced by gripping force, would affect the measurement of deflection of rod. Therefore, if the deflection is used, which is usually captured by a single LVDT, the extra deformation will introduce uncertainty in the experimental results. The use of curvature as measured by the three LVDTs can eliminate the effect.

The CIRFT specifications are as follows:

- Magnetic force-based linear motion: WinTest7 for control and measurement

- Maximum/minimum force of each motor: $\pm 3000 \mathrm{~N}$

- Maximum/minimum displacement of each motor: $\pm 12.7 \mathrm{~mm}$

- U-frame rigid arm length: $101.60 \mathrm{~mm}$

- Specimen gage length $50.8 \mathrm{~mm}$, overall length $152.4 \mathrm{~mm}$, gage diameter, 9.70 to $11.74 \mathrm{~mm}$

- LVDTs for linear motor control: $\pm 12.7 \mathrm{~mm}$ measurement range; $\pm 0.25 \%$ linearity

- Load cells for linear motor control: $\pm 4448 \mathrm{~N}$ measurement range; $\pm 0.02 \%$ linearity

- LVDTs for deflection measurement of specimen: $\pm 5.0 \mathrm{~mm}$ measurement range; $\pm 0.50 \%$ linearity

\subsection{TESTING PROCEDURE}

\subsubsection{Static Testing}

The CIRFT tests a specimen under displacement control with a large amplitude and low ramping rate. The purpose is to develop an understanding of the rod's response under large deformation and identify the bending mechanical properties, including yielding and ultimate strengths and strains.

Ramping is used at a loading rate of $0.1 \mathrm{~mm} / \mathrm{sec}$ and unloading of $0.2 \mathrm{~mm} / \mathrm{sec}$, with a peak displacement of $12 \mathrm{~mm}$. One example of a waveform setup using block mode is as follows, in which TD represents timed data:

- Ramp, Disp 1, $0.1 \mathrm{~mm} / \mathrm{sec}$ to $12 \mathrm{~mm}$, Absolute, TD 180:1800:1

- Ramp, Disp 1, $0.2 \mathrm{~mm} / \mathrm{sec}$ to $0 \mathrm{~mm}$, Absolute, TD not active 
The TD setting is interpreted as scan time: scan points: number of scans: time interval between scans if any.

\subsubsection{Cyclic Testing}

The cyclic test includes cycling and measurements conducted when the cycling is periodically interrupted. The cycling is conducted under load control with low amplitude and high frequency, and the response of the specimen is monitored. The measurements are carried out under displacement control with a small amplitude and low frequency. Figure 1 is a flow chart of the testing process.

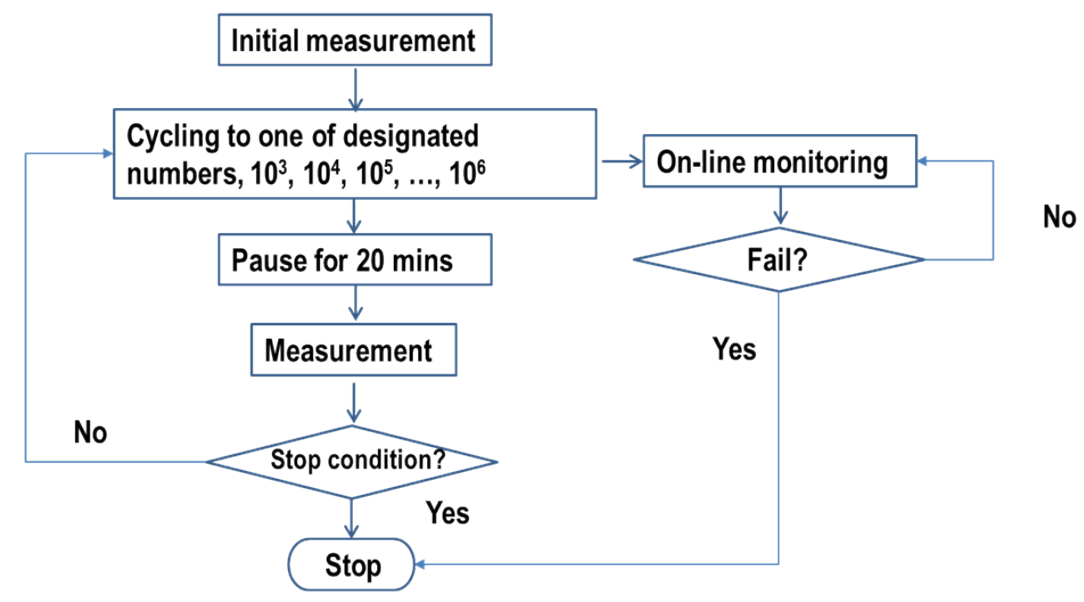

Figure 1. Flowchart illustrating a typical CIRFT process.

The following is an example of a waveform block setup:

- Cyclic indirect, Disp 1, $-100,100 \mathrm{~N}$ at $5 \mathrm{~Hz}$ for 1000 cycles, TD 0.4:400:81:2.5

- Ramp, Disp 1, $5 \mathrm{~mm} / \mathrm{sec}$ to $0 \mathrm{~mm}$, TD not active

The following is an example for measurement with a sine wave:

- Sine, Disp 1, $-0.5 \mathrm{~mm}, 0.5 \mathrm{~mm}$ at $0.05 \mathrm{~Hz}$ for three cycles, TD 70:1400:1

- Ramp, Disp 1, $1 \mathrm{~mm} / \mathrm{sec}$ to $0 \mathrm{~mm}$, TD not active

\subsection{TEST DATA FILES}

The amount of data generated depends on the type of test. Figure 2 illustrates test sessions of a cycle test and the data blocks resulting from monitoring and measurements at each session. In a typical session, the test runs to a designated number of cycles and performs measurements at predetermined amplitudes. The cyclic test stops in the session whenever the predetermined control is reached. 


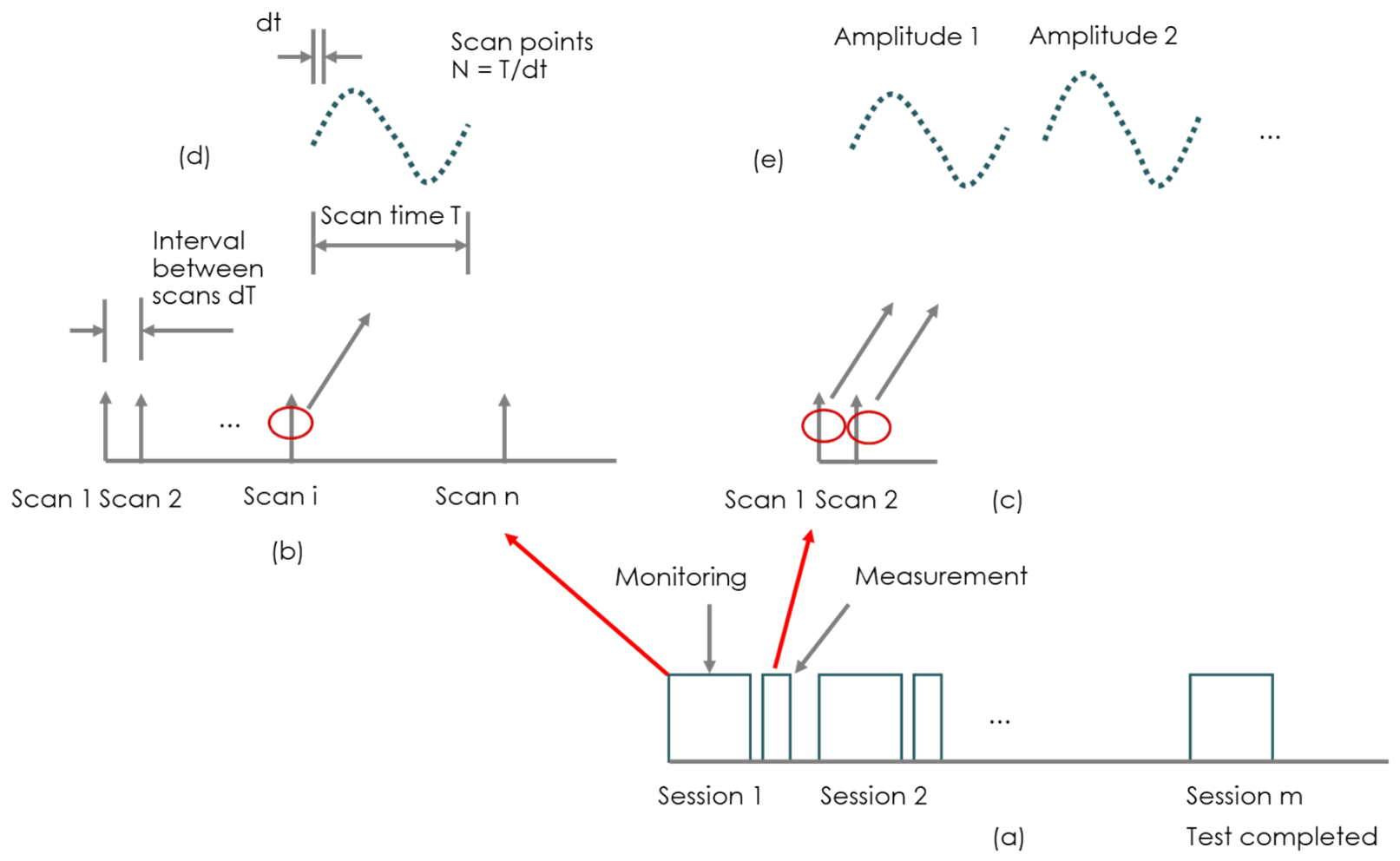

Figure 2. Schematic of scans/data blocks in a typical cyclic test: (a) test sessions, (a) scans of monitoring, (c) scans of measurement, (d) and (e) individual scans/ data blocks.

Bose output files in each session contain a specified number of scans or data blocks as defined in the TD setting, depending on loading conditions. The following examples provide TD settings for a static test and cycling and measurement in a cyclic test, which have same meanings as in Sect. 2.2.1.

- Static data file: $180: 1800: 1$

- Cycling data file: 0.4:400:81:2.5

- Measurement data file: 70:1400:1

The TD file consists of a heading and nine data columns related to the data acquisition channels:

Disp 1-displacement of linear motor $1, \mathrm{~mm}$

Load 1-load of linear motor $1, \mathrm{~N}$

Disp 2-displacement of linear motor 2, $\mathrm{mm}$

Load 2-load of linear motor 2, N

LVDT1-deflection of rod at LVDT $1, \mathrm{~mm}$

LVDT2 - deflection of rod at LVDT 2, mm

LVDT3 - deflection of rod at LVDT 3, $\mathrm{mm}$

Axial $1 \mathrm{cmd}$ - command for linear motor 1 , volt

Axial $2 \mathrm{cmd}$ - command for linear motor 2, volt

\subsection{TECHNICAL ISSUES}

Conventional test systems like WinTest $7^{49}$ usually have limited data processing capabilities, such as waveform display and peak/valley analysis. Such systems cannot satisfy the high-level and high-demand data processing requirements of the SNF CIRFT. 
In particular, the data processing system should at least enable the following functionalities:

- Calculate curvature and moment

- Correct and adjust the measurements as necessary

- Smooth and de-noise the signals

- Extract the mechanical property information for a rod from the acquired data

- Perform necessary statistical analysis of extracted quantities

Although such a system currently is unavailable, many of the functional elements have been developed using MATLAB in the SNF vibration integrity project. The data processing package has been used extensively in data processing of CIRFT results for spent fuels from various reactors, including HB Robinson, Limerick, North Anna, mixed oxide fuels, and sister rods.

This report describes the main components of the package. 


\section{TECHNICAL APPROACH}

\subsection{LANGUAGE AND TOOLBOX}

The CIRFT data processing package is developed using the description language MATLAB. The programs or MATLAB functions are saved in mfiles (*.m). Each mfile, in comment lines (beginning with $\%$ ), has a brief description on the functionality of function and an example showing how to run the function in the command line.

The example is generally self-explained in terms of input and output. However, this report also describes the main components of input and output of each function when it is necessary for clarification.

The data processing package needs the Curve Fitting ToolBox for support.

\subsection{BASIC FUNCTIONS}

\subsubsection{Curvature Calculation}

The curvature of a bending rod is calculated based on the three deflections measured by three LVDTs, as shown in Figure 3. The spacing of these LVDTs is denoted as $h$ in Figure $3(12 \mathrm{~mm})$. As discussed previously, ${ }^{31,34,35,37-39}$ the LVDTs use a disc-shaped probe head with a diameter of $6 \mathrm{~mm}$. The size of the discs is comparable to the sensor distance; therefore, their effect on the calculation needs to be considered.

mfiles: thrpts.m, thrpts4.m

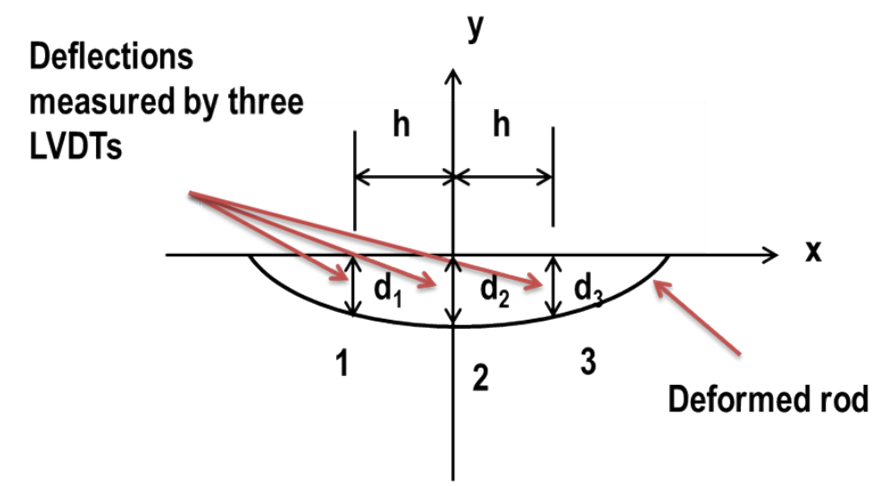

Figure 3. Diagram of three deflections used in curvature calculation where sensor spacing is $h$.

\subsubsection{Sensor Spacing Correction}

The contact of the probe heads affects the spacing $h$, depending the sign of the curvature, as shown in Figure 4 . When the rod is being bent, the contact mode of the probe disc against the rod changes from line contact into point contact, and the contact point is located outside the edge of the disc in positive bending and inside the edge of the disc in negative bending. The change in the contact modifies the sensor spacing $h$ by the amount of $\Delta h$. Two approaches to correcting the spacing have been developed: a calibration rod and self-correction. 


\subsubsection{Calibration rod for spacing correction}

This method uses an elastic rod with a known flexural rigidity. The calibration rod is tested under an elastic condition with a loading range equivalent to that in the application. The rigidity of the calibration rod is thus known because the Young's modulus and moment of inertia are given. Therefore, for a moment applied, the correction of sensor spacing can be achieved by comparing the curvature based on the LVDTs with the calculated curvature.

This method is used in data analysis of static testing.

mfile: curvacycle $3 . m$

\subsubsection{Self- correction}

The gage length of a specimen is considered to be constant whether it is in a positive or a negative bending direction. For reversed bending, the correction introduced to the spacing on the positive and negative sides of the bending shall result in an identical gage length. This approach has been confirmed by calibration rods using several measurement methods, including chisel typical probe, strain gage, and moment-based curvature at various loading amplitudes and frequencies.

This method is used in analyzing the data of a cycle test. The gradual correction of spacing is shown in Figure 5, along with the effect on the curvature profile.

mfile: defcurv1.m

(a)

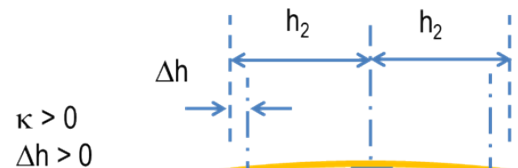

(b)

$$
\Delta \mathrm{h}>0
$$
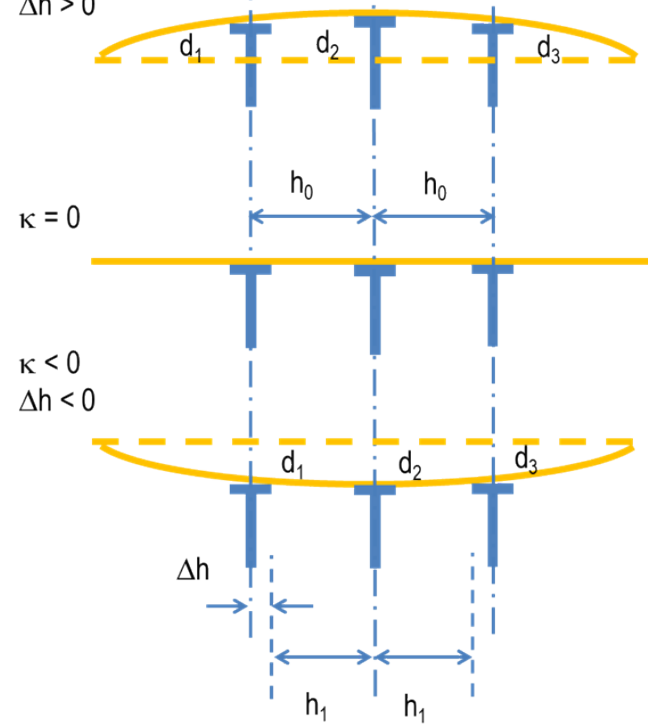

Figure 4. Diagram of curvature corrections when linear motors are in (a) pull, (b) neutral position, and (c) push. When rod is being bent, the contact mode of the probe disc with the rod changes from line contact into point contact, and the contact point is located outside the edge of the disc in positive bending and inside the edge of the disc in negative bending. 


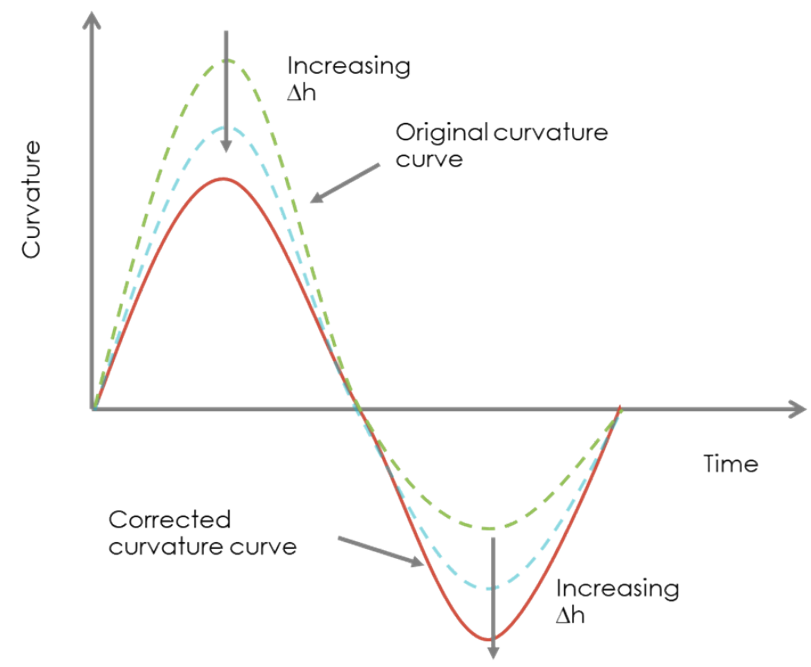

Figure 5. A schematic showing the spacing correction applied to correct the curvature and adjust the curvature profile.

\subsubsection{Dynamic Amplification}

It has been shown that the outside fiber strain of a bending rod with a curvature measured by three LVDTs is higher than that measured using a strain gage. ${ }^{42,43}$ The amplification is perhaps related to the dynamic response of the measurement system. A reduction factor is applied to offset this effect, which depends on driving frequency.

mfile: defcurv1.m

\subsubsection{Frictional Resistance}

The frictional resistance of the U-frame system becomes significant when the displacement is large and thus needs to be considered in static testing. This effect is evaluated by using an empty run in which the test is conducted without a specimen loaded. Thereafter, the resistance is taken from the readings of the load channels. Figure 6 shows the procedure adopted.

mfile: read4cal1.m

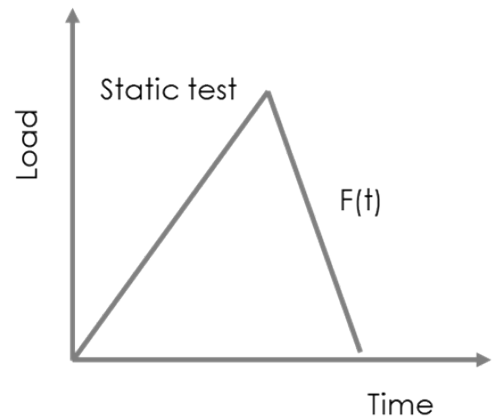

(a)

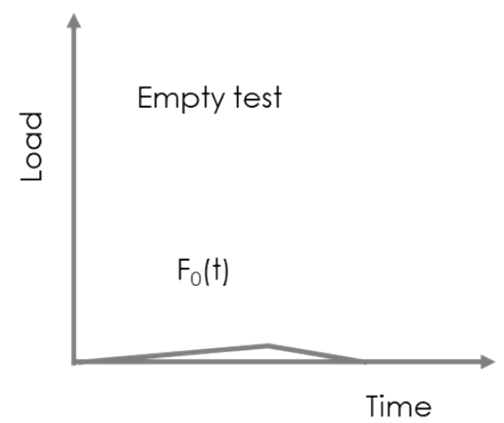

(b)

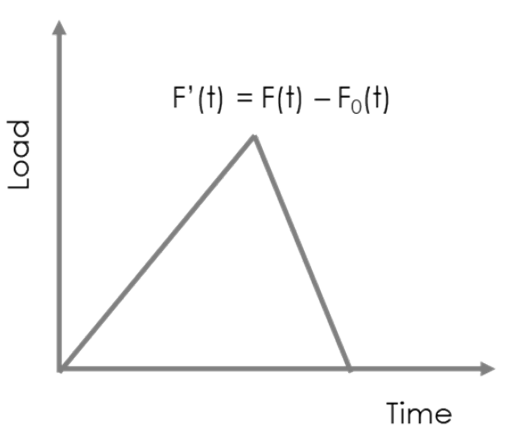

(c)

Figure 6. Schematic showing the load profiles of (a) a static test, (b) an empty test, and (c) a test with the effect of frictional resistance removed. 


\subsubsection{De-noising}

Two methods are used for removing noise from signals.

1. Smoothing using spline functions

For static test data and measurement data, spline functions selected from the MATLAB Curve Fitting Toolbox are used.

mfiles: curvacycle $3 . \mathrm{m}$, read4cal41.m

2. De-noising

For periodic signals in cyclic testing, fast Fourier transform (FFT) is used to extract the fundamental harmonics of curvature signals, as shown in Figure 7. The denoised signals are used in the subsequent characterization and evaluation.

mfile: defcurv1.m
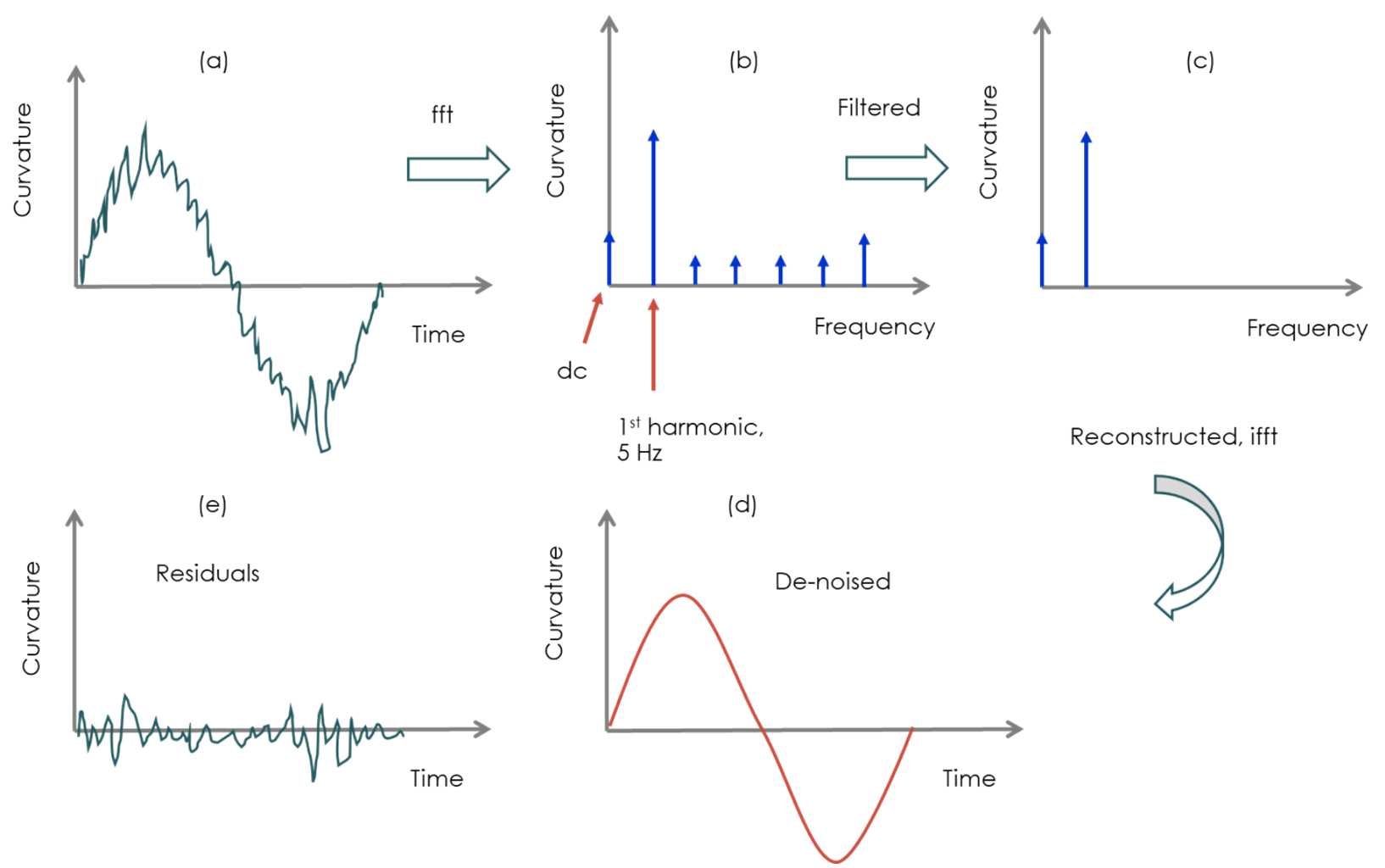

Figure 7. Schematic to illustrate the procedure used to remove noise in curvature data: (a) raw signal, (b) linear spectrum, (c) spectrum after filtering, (d) signal reconstructed, and (e) residuals.

\subsection{MODULES OF PACKAGE}

The data processing package for CIRFT consists of four modules, each with a specific objective. The relationship of these modules is shown in Figure 8. Each module is composed of functions, as described in the following sections. 


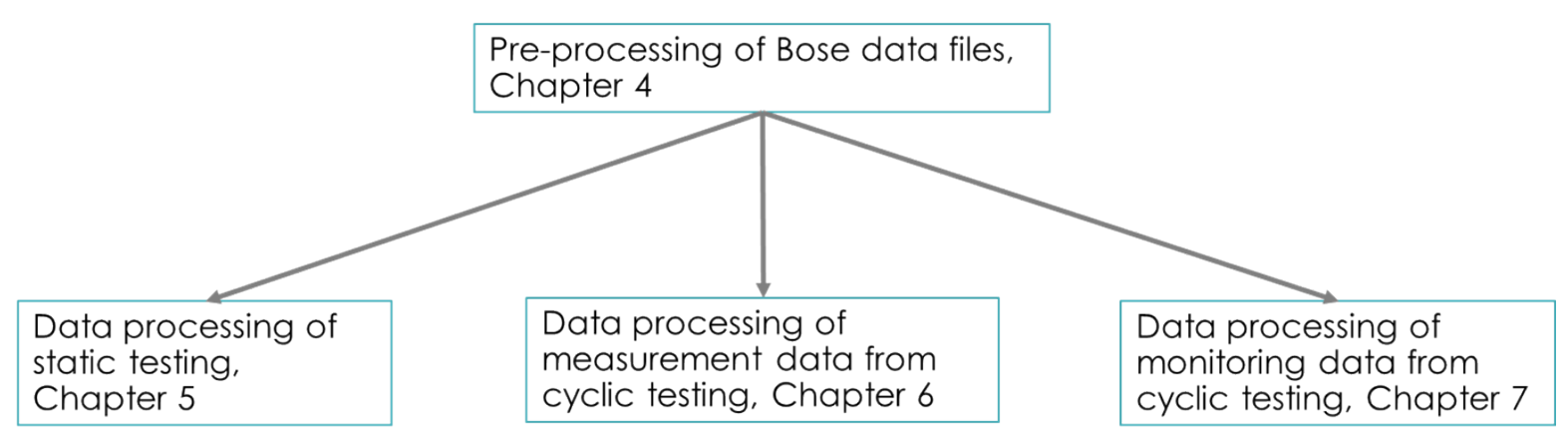

Figure 8. Modules for data processing.

\subsection{GENERAL FLOWCHART OF DATA PROCESSING}

Figure 9 is a general flowchart for data processing procedures in a typical data analysis. The following sections discuss all these procedures with mfiles, input, output, and necessary technical details.

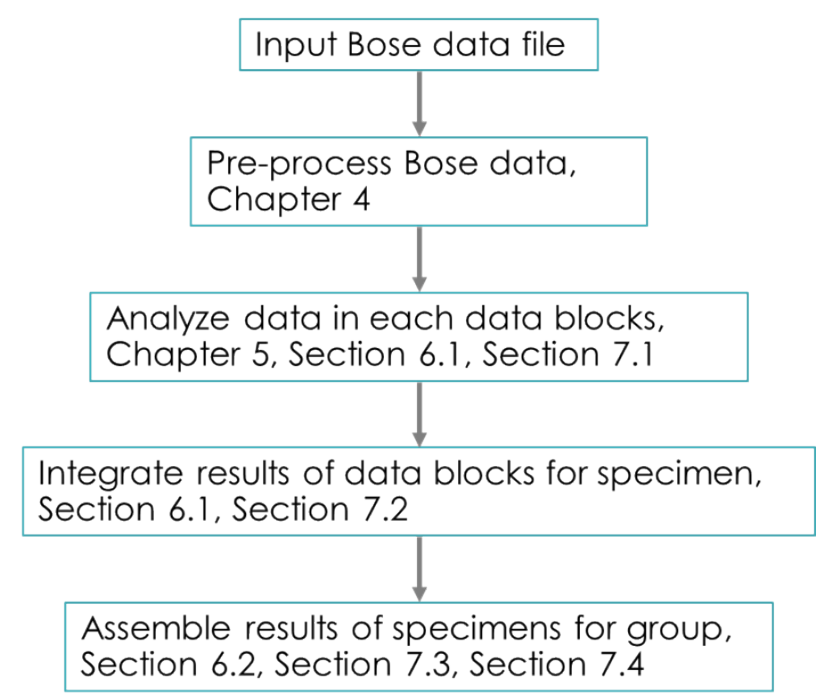

Figure 9. General data processing for a CIRFT data set. 


\section{PRE-PROCESSING}

\subsection{DATA STRUCTURE}

CIRFT data are structured with four layers, as shown in Figure 10, from the group level down to scans or data blocks.

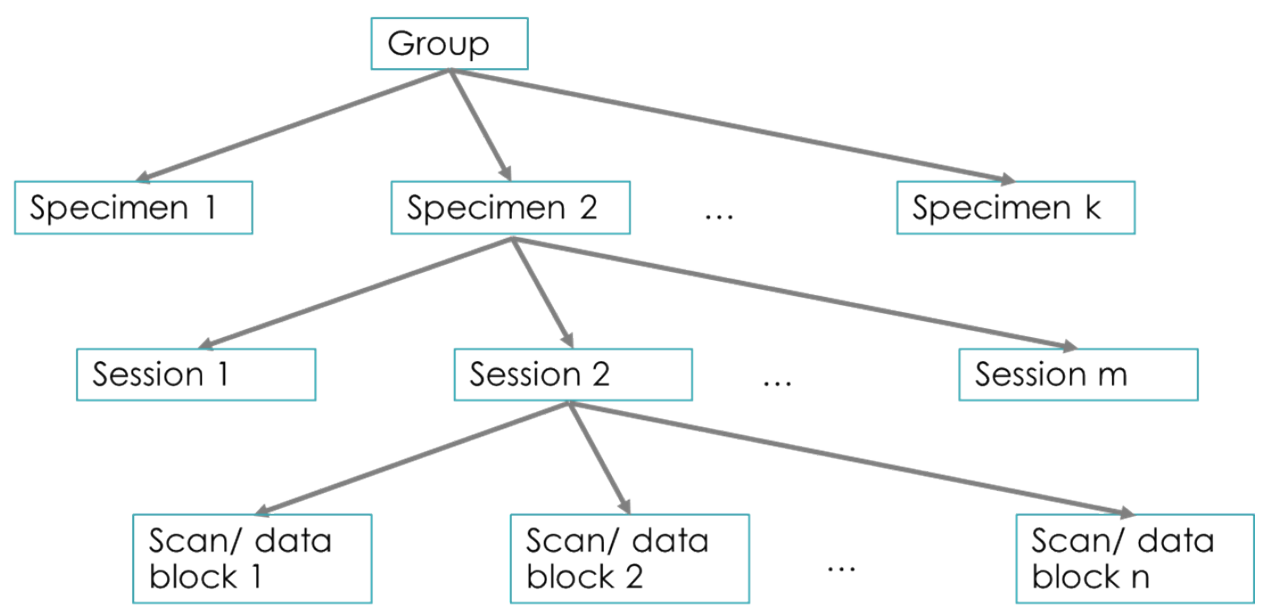

Figure 10. Architecture of CIRFT data structure.

\subsection{TEST CONDITION SUMMARY DATA SHEETS}

\subsubsection{Test Summary for Individual Specimens}

A data sheet is prepared to summarize information for the tests conducted on a specimen, covering measurements and cycling. An example is given in Table 1 for a specimen under cyclic testing. The data sheet includes columns for test date, test number, peak 1 (pk1, valley), peak 2 (pk2, peak), driving frequency, cycles in the test, accumulated cycles, LVDT reset, and notes. The data sheet serves as input to initiate the data conversion.

Table 1. Example of data sheet summarizing tests on a specimen.

\begin{tabular}{|c|l|l|l|l|r|r|r|l|}
\hline Date & \multicolumn{1}{|c|}{ No. } & \multicolumn{1}{c|}{$\begin{array}{c}\text { pk1 (mm, } \\
\mathbf{N})\end{array}$} & $\begin{array}{c}\mathbf{p k 2} \\
\mathbf{( m m}, \mathbf{N})\end{array}$ & Freq (Hz) & Cycles & \multicolumn{1}{c|}{ Sum } & Reset & Notes \\
\hline $8 / 26 / 2019$ & 1 & -0.4 & 0.4 & 0.05 & 3 & 1 & 1 & \\
\hline $8 / 26 / 2019$ & 2 & -0.6 & 0.6 & 0.05 & 3 & 1 & 1 & \\
\hline $8 / 26 / 2019$ & 3 & -100 & 100 & 5 & 1000 & 1000 & 1 & \\
\hline $8 / 26 / 2019$ & 4 & -0.4 & 0.4 & 0.05 & 3 & 1000 & 1 & \\
\hline $8 / 26 / 2019$ & 5 & -0.6 & 0.6 & 0.05 & 3 & 1000 & 1 & \\
\hline $8 / 26 / 2019$ & 6 & -100 & 100 & 5 & 2453 & 3453 & 0 & Failed \\
\hline
\end{tabular}

\subsubsection{Test and Specimen Status Summaries for Group}

Summary data sheets for a group of specimens generally provide the material and testing conditions of each specimen, including data acquisition. 
An example for CIRFT conditions is shown in Table 2, with part of a data sheet included. It includes columns for data directory (DDR), specimen ID, fuel segment ID, rod inside diameter (ID), rod outside diameter (OD), pellet diameter (PD), hydrogen content, load amplitude (DP/2), moment amplitude $(\mathrm{DM} / 2)$, number of cycles to failure or completion, failure confirmation, follow-up test confirmation, and sets of measurements (number of interruptions for measurements).

Table 2. Summary of CIRFT process for a specific fuel.

\begin{tabular}{|c|c|c|c|c|c|c|c|c|c|c|c|c|}
\hline $\begin{array}{c}\text { DD } \\
\text { R }\end{array}$ & $\begin{array}{c}\text { Spec } \\
\text { ID }\end{array}$ & Seg ID & $\begin{array}{l}\text { ID, } \\
\text { mm }\end{array}$ & $\begin{array}{l}\text { OD, } \\
\text { mm }\end{array}$ & $\begin{array}{l}\text { PD, } \\
\text { mm }\end{array}$ & $\begin{array}{c}\text { H, } \\
\text { ppm }\end{array}$ & $\begin{array}{c}\mathrm{DP} / 2, \\
\mathrm{~N}\end{array}$ & $\begin{array}{c}\mathrm{DM} / 2, \\
\mathrm{Nm}\end{array}$ & $\begin{array}{c}\text { \# of } \\
\text { cycles }\end{array}$ & Failure & $\begin{array}{c}\text { Follow } \\
\text {-up }\end{array}$ & $\begin{array}{c}\text { Meas. } \\
\text { sets }\end{array}$ \\
\hline D2 & D2 & $\begin{array}{l}\text { 30AD05-2050- } \\
2203\end{array}$ & 8.34 & 9.43 & 8.34 & 0 & 60 & 6.10 & $\begin{array}{l}1.33 \mathrm{E}+0 \\
5\end{array}$ & 1 & 0 & 4 \\
\hline D1 & D1 & $\begin{array}{l}\text { 30AD05-2630- } \\
2783\end{array}$ & 8.34 & 9.43 & 8.34 & 0 & 120 & 12.19 & $\begin{array}{l}2.23 \mathrm{E}+0 \\
4\end{array}$ & 1 & 0 & 3 \\
\hline $\mathrm{S} 2$ & S2 & $\begin{array}{l}\text { 30AE14-672- } \\
825\end{array}$ & 8.34 & 9.46 & 8.34 & 0 & 175 & 17.78 & $\begin{array}{l}1.63 \mathrm{E}+0 \\
3\end{array}$ & 1 & 1 & 0 \\
\hline D3 & D3 & $\begin{array}{l}\text { 30AE14-2850- } \\
3003\end{array}$ & 8.34 & 9.46 & 8.34 & 0 & 120 & 12.19 & $\begin{array}{l}9.80 \mathrm{E}+0 \\
3\end{array}$ & 1 & 0 & 2 \\
\hline D4 & D4 & $\begin{array}{l}\text { 30AE14-3156- } \\
3309\end{array}$ & 8.34 & 9.46 & 8.34 & 0 & 60 & 6.10 & $\begin{array}{l}1.13 \mathrm{E}+0 \\
5\end{array}$ & 1 & 0 & 4 \\
\hline S3 & S3 & $\begin{array}{l}\text { 3A1F05-1853- } \\
2006\end{array}$ & 8.42 & 9.46 & 8.42 & 0 & 175 & 17.78 & $\begin{array}{l}1.30 \mathrm{E}+0 \\
3\end{array}$ & 1 & 1 & 0 \\
\hline D12 & D12 & $\begin{array}{l}\text { 3A1F05-2025- } \\
2178\end{array}$ & 8.42 & 9.46 & 8.42 & 0 & 100 & 10.16 & $\begin{array}{l}4.82 \mathrm{E}+0 \\
4\end{array}$ & 1 & 0 & 3 \\
\hline D9 & D9 & $\begin{array}{l}\text { 3A1F05-3214- } \\
3367\end{array}$ & 8.42 & 9.46 & 8.42 & 0 & 100 & 10.16 & $\begin{array}{l}3.45 \mathrm{E}+0 \\
3\end{array}$ & 1 & 0 & 2 \\
\hline D14 & D14 & $\begin{array}{l}\text { 3A1F05-3367- } \\
3520\end{array}$ & 8.42 & 9.46 & 8.42 & 0 & 50 & 5.08 & $\begin{array}{l}2.14 \mathrm{E}+0 \\
5\end{array}$ & 1 & 0 & 4 \\
\hline D8 & D8 & $\begin{array}{l}\text { 3D8E14-1178- } \\
1331\end{array}$ & 8.38 & 9.49 & 8.38 & 0 & 60 & 6.10 & $\begin{array}{l}2.12 \mathrm{E}+0 \\
5\end{array}$ & 1 & 0 & 4 \\
\hline D7 & D7 & $\begin{array}{l}\text { 3D8E14-2412- } \\
2565\end{array}$ & 8.38 & 9.49 & 8.38 & 0 & 60 & 6.10 & $\begin{array}{l}1.91 \mathrm{E}+0 \\
5\end{array}$ & 1 & 0 & 4 \\
\hline$\cdots$ & & & & & & & & & & & & \\
\hline
\end{tabular}

An example for CIRFT specimen condition is shown in Table 3, with part of a data sheet included. It includes columns for specimen ID (SID), rod segment ID (RID), lower elevation of segment (LE) in mm, pellet length (PL) in mm, cladding wall thickness (CTK) in $\mu \mathrm{m}, \mathrm{BU}$ in $\mathrm{GWd} / \mathrm{MTU}$, zone number, cladding type (CLD), status of specimen (STT), and follow-up confirmation. 
Table 3. Specimen condition used in CIRFT testing.

\begin{tabular}{|l|l|l|l|l|l|l|l|l|l|}
\hline \multicolumn{1}{|c|}{ SID } & \multicolumn{1}{|c|}{ RID } & \multicolumn{1}{c|}{ LE } & \multicolumn{1}{c|}{ PL } & CTK & BU & Zone & \multicolumn{1}{c|}{ CLD } & \multicolumn{1}{c|}{ STT } & $\begin{array}{c}\text { Follow- } \\
\text { up }\end{array}$ \\
\hline D2 & 30AD05 & 2050 & 10.26 & 543 & 52 & zone1 & M5 & AR & 0 \\
\hline D1 & 30AD05 & 2630 & 10.26 & 543 & 52 & zone1 & M5 & AR & 0 \\
\hline S2 & 30AE14 & 672 & 10.28 & 560 & 52 & zone1 & M5 & HS & 1 \\
\hline D3 & 30AE14 & 2850 & 10.28 & 560 & 52 & zone1 & M5 & HT & 0 \\
\hline D4 & 30AE14 & 3156 & 10.28 & 560 & 52 & zone1 & M5 & HT & 0 \\
\hline S3 & 3A1F05 & 1853 & 9.92 & 520 & 50 & zone1 & LT & ST & 1 \\
\hline D12 & 3A1F05 & 2025 & 9.92 & 520 & 50 & zone1 & LT & AR & 0 \\
\hline D9 & 3A1F05 & 3214 & 9.92 & 520 & 50 & grid6 & LT & AR & 0 \\
\hline D14 & 3A1F05 & 3367 & 9.92 & 520 & 50 & zone2 & LT & AR & 0 \\
\hline D8 & 3D8E14 & 1178 & 10.05 & 555 & 55 & grid2 & ZL & AR & 0 \\
\hline D7 & 3D8E14 & 2412 & 10.05 & 555 & 55 & zone1 & ZL & AR & 0 \\
\hline$\ldots$ & & & & & & & & & \\
\hline
\end{tabular}

An example for CIRFT data acquisition conditions is shown in Table 4, with part of a data sheet included. It includes columns for specimen ID, data acquisition status, and notes.

Table 4. Data acquisition condition of CIRFT testing.

\begin{tabular}{|l|l|l|}
\hline \multicolumn{1}{|c|}{ Specimen ID } & \multicolumn{1}{c|}{ Data acquisition } & \multicolumn{1}{c|}{ Notes on data acquisition system } \\
\hline D2 & 0 & No issue \\
\hline D1 & 0 & No issue \\
\hline S2 & 0 & No issue \\
\hline D3 & 0 & No issue \\
\hline D4 & 0 & No issue \\
\hline S3 & 0 & No issue \\
\hline D12 & 0 & No issue \\
\hline D9 & 0 & No issue \\
\hline D14 & 0 & No issue \\
\hline D8 & 0 & No issue \\
\hline D7 & 1 & Disp/defl in question \\
\hline$\ldots$ & & \\
\hline
\end{tabular}

\subsection{DATA CONVERSION PREPARATION}

Data conversion extracts relevant data signals from the Bose output files using the following steps.

1. Create data directory for each specimen: ddr

2. Create subdirectory: ddrlbose, and copy Bose output files into this subdirectory

3. Call bose2txt.m to extract signals and save data to ddr $\mid$ bose $\left.\right|^{*}$.dat 


\subsection{1 bose2txt.m}

function bose2txt(ddr,iffigure, ifprint);

$\% \quad$ mfile: bose2txt.m

$\% \quad$ convert Bose output into text files

$\% \quad$ example: close all; clear all; bose2txt('D9',0,0);

$\%$ functions called: readbose $01 . \mathrm{m}$

\subsection{2 readbose01.m}

function readbose01(ddr, testnum, iffigure, ifprint);

$\% \quad$ mfile: readbose $01 . m$

$\%$ read from Bose

$\%$ example:

$\% \quad$ readbose01('MOX13','test001 08172015012444 tddf.TXT', 1, 0);

\subsection{DECOMPOSITION OF MEASUREMENT DATA}

File decomposition separates a file with multiple data blocks into single- data block files for next-step analysis. The following general steps are used for the measurement of data decomposition, where * or ???? is a space holder for specimen.

1. Referring to Table 1, edit input file and save into ddr ${ }^{*}$.inp

2. Call MeasFDecomp.m

a. to decompose Bose output into individual data blocks and save data into \ddr and

b. save general summary sheet into measurement sheet and save next-step-input file into ddrlmeas????.inp

\subsubsection{MeasFDecomp.m}

function MeasFDecomp(ddr, testsum, fl4rgd, ifprint);

$\% \quad$ mfile: MeasFDecomp.m

$\%$ decompose measurement data to data blocks

$\%$ example:

$\% \quad$ close all; clear all; MeasFDecomp('MOX13','mox13.inp','measmox13.inp',0);

$\%$ functions called: num22str.m, readbose12.m

Input:

$\mathrm{D}^{*}$.inp is given as an example.

$\begin{array}{llllllll}8 / 26 / 2019 & 1 & -0.4 & 0.4 & 0.05 & 3 & 1 & 1 \\ 8 / 26 / 2019 & 2 & -0.6 & 0.6 & 0.05 & 3 & 1 & 1 \\ 8 / 26 / 2019 & 3 & -100 & 100 & 5 & 1000 & 1000 & 1 \\ 8 / 26 / 2019 & 4 & -0.4 & 0.4 & 0.05 & 3 & 1000 & 1 \\ 8 / 26 / 2019 & 5 & -0.6 & 0.6 & 0.05 & 3 & 1000 & 1 \\ 8 / 26 / 2019 & 6 & -100 & 100 & 5 & 2453 & 3453 & 0\end{array}$


Output:

measd*.inp of sister rods $\mathrm{D}^{*}$ is given below as an example, including measurement set number, test number, measurement amplitudes in each set, number of cycles completed when measurement is made, whether LVDT reset is renewed.

$\begin{array}{ccccc}1 & \text { test00101 } & 0.4 & 1 & 1 \\ 1 & \text { test00201 } & 0.6 & 1 & 1 \\ 2 & \text { test00401 } & 0.4 & 1000 & 1 \\ 2 & \text { test00501 } & 0.6 & 1000 & 1\end{array}$

\subsection{2 num22str.m}

function ns $=$ num22str(num)

$\% \quad$ mfile: num22str.m

$\% \quad$ append zeros

\subsection{3 readbose12.m}

function readbose12(ddr, testfile, LS, NP, NS, ifprint)

$\% \quad$ mfile: readbose $12 . m$

$\% \quad$ testfile- export from WinTest7

$\% \quad$ LS - length of scan, sec

$\% \quad \mathrm{NP}$ - \# of points in a scan

$\% \quad$ NS- \# of scans in the export

$\%$ example:

$\% \quad$ readbose12('MOX13','test001.dat', 70, 1400, 1,0)

\subsection{DECOMPOSITION OF MONITORING DATA}

File decomposition separates a file with multiple data blocks into files with single data blocks for nextstep analysis. The following general steps are used for the monitoring data decomposition.

1. Make directory 'ldcs' in \CIRFT for readbose122.m plot output

2. Call MonFDecomp

a. to decompose Bose output into individual data blocks and save data into \ddr and

b. save general summary sheet into measurement sheet and save next-step-input file into ddr\mon????.inp

\subsubsection{MonFDecomp.m}

function MonFDecomp(ddr, testsum, fl4rgd, iffigure, ifprint)

$\% \quad$ mfile: MonFDecomp.m

$\%$ decompose monitoring data to data blocks

$\%$ example:

$\% \quad$ close all;clear all;MonFDecomp('MOX13','mox13.inp','monmox13.inp',1,0);

$\%$ functions called: num22str.m, readbose122.m 


\section{Output:}

mon*.inp is given below as an example, including test number, frequency, starting block, block number increment, final block, number of cycles completed in test.

$\begin{array}{cccccc}\text { test003 } & 5 & 8 & 1 & 80 & 1 \\ \text { test006 } & 5 & 2 & 1 & 20 & 1000\end{array}$

\subsection{2 readbose122.m}

function readbose122(ddr, testfile, LS, NP, NS, reset_renew, ifprint)

$\% \quad$ mfile: readbose $121 . \mathrm{m}$

$\%$ decompose export from WinTest7

$\% \quad$ LS - length of scan, sec

$\% \quad \mathrm{NP}$ - \# of points in a scan

$\% \quad$ NS- \# of scans in the export

$\% \quad$ Example:

$\% \quad$ readbose122('D13','test003.dat', 0.4, 400, 80, 1, 0)

$\% \quad$ functions called: Base_level_def.m

Input:

reset_renew—control of new base level of LVDTs

\subsubsection{Base_level_def.m}

function $[\mathrm{fct} 10, \mathrm{fct} 20, \mathrm{fct} 30]=$ Base_level_def(t, fct1, fct $2, \mathrm{fct} 3, \mathrm{ttltxt}$, iffigure $)$;

$\% \quad$ least square spline approximation, cubic spline with 10 pieces for 2 periods

$\%$ define baseline for LVDTs

$\% \quad$ toolbox required: Curve Fitting 


\section{DATA PROCESSING FOR STATIC TESTING}

There are several tasks in the data processing of static tests:

- Correct the sensor spacing in the curvature calculation and remove the frictional load from the load channels.

- Obtain characteristic points and bending mechanical properties.

- Integrate the data sets of individual specimens in the group for the report.

The package provides four main functions to meet the requirements, curvacycle $3 . \mathrm{m}$, mntcurv.m, meascol4.m, and curv3col.m, as shown in the flow chart in Figure 11.

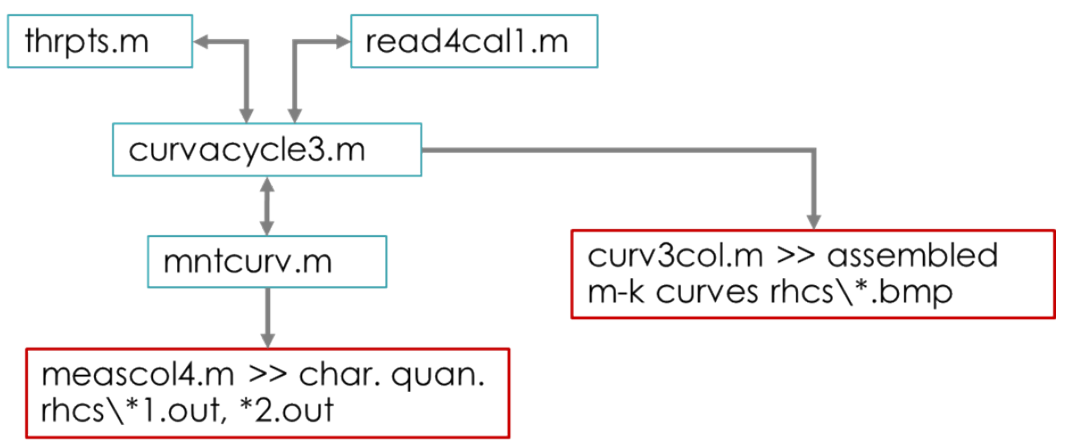

Figure 11. Flowchart of data processing for static test.

\subsection{SPECIMEN MOMENT-CURVATURE CURVES}

The following four steps make up the data processing procedure to obtain the moment-curvature curve and properties. The comment lines of the relevant functions and main input are provided in the subsequent subsections.

1. Run readbose $01 . \mathrm{m}$ and readbose12.m to extract data from Bose files; if not yet,

2. Run curvacycle 3.m to get moment and curvature data

3. Prepare sta*.inp

4. Run mntcurv.m to obtain characteristics of moment-curvature curve

\subsection{1 curvacycle3.m}

function [mss_t, mss_m, kss_k, ms, ks] = curvacycle3(sp, testnum, testnum0, ifcor, dh, iffigure, ifprint)

$\% \quad$ mfile: curvacycle $3 . m$

$\%$ dataflow: readbose01-> readbose12-> read4cal1-> curvacycle1-> reductdat1-> dkdmcol

$\% \quad$ calculate curvature, correct, and smooth data

$\%$ example:

$\% \quad$ [mss_t, mss_m, kss_k, ms, ks] = curvacycle3('S1','test00101','test00001', 1, 2.5, 1, 1);

$\% \quad$ functions called: thrpts.m, read4cal1.m

$\% \quad$ toolbox required: Curve Fitting

Input: 
testnum0 - static data from empty run for friction resistance evaluation. The empty test generally has the same data acquisition setting as the real static test.

$\mathrm{dh}$ - sensor spacing correction

\subsection{2 read4cal1.m}

function [t, dsp, load, mmnt, flct1, flct2, flct3, ttltxt] = read4cal1(sp, testnum, testnum0, iffigure, ifprint);

$\% \quad$ mfile: read4cal1.m

$\% \quad$ read data block, truncate data, remove frictional force, cal. moment

$\%$ dataflow: readbose $01->$ readrose $12->$ read4cal-> curvacycle- $>$ reductdat $1->$ dkdmcol

$\% \quad$ Example:

$\% \quad$ [ts, ds, ps, ms, fls, f2s, f3s, ttltxt] =read4cal1('test00201','test00101',1, 0);

\subsection{3 thrpts.m}

function $[x, y, R, k]=\operatorname{thrpts}(d 1, d 2, d 3, h)$;

$\% \quad$ mfile: thrpts.m

$\%$ get curvature as the inverse of radius using three-point circle equation

$\%$ example:

$\% \quad[\mathrm{x}, \mathrm{y}, \mathrm{R}, \mathrm{k}]=\operatorname{thrpts}(0.1337,0.139,0.127,12)$

\subsection{4 mntcurv.m}

function mntcurv(sp, testnum, inpfil)
$\% \quad$ mfile: mntcurv.m
$\% \quad$ find curve fitting parameters and interaction points
$\%$ example:
$\% \quad$ close all; clear all; mntcurv('S1','test00101','stasis.inp');
$\% \quad$ functions called: polysigm.m, findpnt.m

The following (see Figure 12) are the steps in mntcurv.m to characterize a static moment-curvature curve:

1. Divide the curve into loading/unloading stages using the peak moment.

2. Pick the feature points: $\mathrm{p} 1$ — starting; $\mathrm{p} 2$ — turning to the second linear segment; $\mathrm{p} 3$ - turning to the quadratic; $\mathrm{p} 4$ - the peak point.

3. Obtain the fitting parameters of four segments by calling polysigm.m: p1-p2, p2-p3, p3-p4, p4-end.

4. Obtain the coordinates of the interaction points: A - between the two linear segments (xi1, yi1); Bbetween the second linear and quadratic (xi2, yi2); $\mathrm{C}$ - the intercept of the unloading line with the loading part at $0.2 \%$ equivalent strain (xi5, yi5); D — peak point (xi3, yi3); E-intercept of the unloading at $\mathrm{x}$-axis (xi4, yi4).

Input:

sta*.inp is given as an example. It includes columns dds, sps, sgids, ids, ods, pdias, hc, tests, test0s 
Output:

*.out in $\backslash$ ddr. The array consists of curve fitting parameters (9) at the four segments, coordinates (10) at the five interaction points.

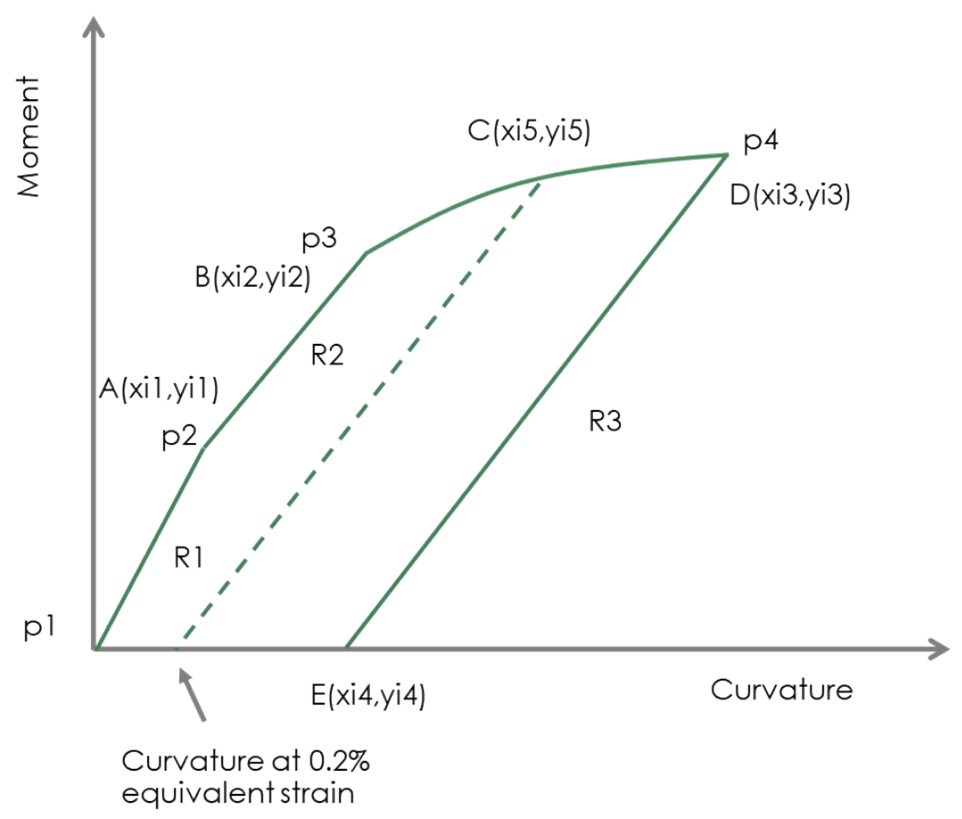

Figure 12. A schematic showing the quantities used in characterization.

\subsection{5 polysigm.m}

function [pmt, xstar, ystar] = polysigm(epsn, sigm, n, yi, epsns);

$\% \quad$ mfile: polysigm.m

$\% \quad$ curve fit for the segment that is defined by yi using $\mathrm{n}$ polynomial

\subsection{6 findpnt.m}

function $[x i, y i]=$ findpnt $(x 1, y 1, x 2, y 2)$;

$\% \quad$ mfiles: findpnt.m

$\% \quad$ find intersection point if lines are defined by $\mathrm{x} 1, \mathrm{y} 1, \mathrm{x} 2, \mathrm{y} 2$

\subsection{ASSEMBLED CURVES FOR TEST GROUP}

Data integration for a group of specimens includes the characteristic points and properties and the moment vs. curvature curves or the equivalents. The data processing procedure is as follows.

1. Create subdirectory 'rhcs' in $\backslash$ CIRFT.

2. Run meascol4.m to output static testing results to 'rhcs.' 
3. Run curv3col.m to assemble curves for the group and save plots to 'rhcs.'

\subsection{1 meascol4.m}

function meascol4(inpfil)

$\% \quad$ mfile: meascol4.m

$\% \quad$ objective: to assemble characteristic quantities

$\% \quad$ example: close all; clear all; meascol4('stasis.inp')

Output:

sta*1.out

hc-hydrogen content

EI1 - rigidity in the first linear segment

EI2 - rigidity in the second linear segment

EI3 - rigidity in the unloading stage (segment)

$\mathrm{ka}$ - curvature at $\mathrm{A}$, intersection between the first and second linear segments

$\mathrm{kb}$ - curvature at $\mathrm{B}$, intersection between the second linear and quadratic segments

$\mathrm{kc}$ - curvature at $\mathrm{C}, 0.2 \%$ equivalent strain

$\mathrm{kd}$ - curvature at $\mathrm{D}$, peak moment

ke-curvature at E, residual curvature when the moment returns to zero

ma-moment at A, intersection between the first and second linear segments

$\mathrm{mb}$ - moment at $\mathrm{B}$, intersection between the second linear and quadratic segments

mc-moment at $\mathrm{C}, 0.2 \%$ equivalent strain

md-moment at $\mathrm{D}$, peak moment

me- moment at E, or zero

sta*2.out

hc-hydrogen content

E1-equivalent elastic modulus in the first linear segment

E2 - equivalent elastic modulus in the second linear segment

E3 - equivalent elastic modulus in the unloading stage (segment)

ea-equivalent strain at $\mathrm{A}$, intersection between the first and second linear segments

$\mathrm{eb}$ - equivalent strain at $\mathrm{B}$, intersection between the second linear and quadratic segments

ec-equivalent strain at $\mathrm{C}, 0.2 \%$ equivalent strain

ed-equivalent strain at $\mathrm{D}$, peak moment

ee- equivalent strain at $\mathrm{E}$, residual equivalent strain when the equivalent stress returns to zero

sa - equivalent stress at $\mathrm{A}$, intersection between the first and second linear segments

$\mathrm{sb}$ - equivalent stress at $\mathrm{B}$, intersection between the second linear and quadratic segments

sc-equivalent stress at $\mathrm{C}, 0.2 \%$ equivalent strain

sd-equivalent stress at $\mathrm{D}$, peak moment

se- equivalent stress at $\mathrm{E}$, or zero

\subsection{2 curv3col.m}

function [lghnds, lgtxts] = curv3col(inpfile, pltid, fig4);

$\% \quad$ Stress vs strain

$\% \quad$ assemble curves of moment vs curvature or equivalent stress vs. strain 
$\% \quad$ example: close all; clear all; [lghnds, lgtxts]= curv3col('stasis.inp', 1, 0); 


\section{DATA PROCESSING FOR MEASUREMENT}

Measurement data processing proceeds at the specimen level and group level. Data for individual specimens are processed in each data block and then re-integrated to the session or interruption. As can be seen, the Bose output at each session is composed of a single data block. Multiple outputs are usually obtained at multiple measurement amplitudes. For a group of specimens, characterization quantities and properties will be extracted and/or assembled in terms of fatigue curves and scatter plots.

Figure 13 provides an overview of functions and relations among them. The comment lines of each function and supporting sub-functions are provided in the following subsections.

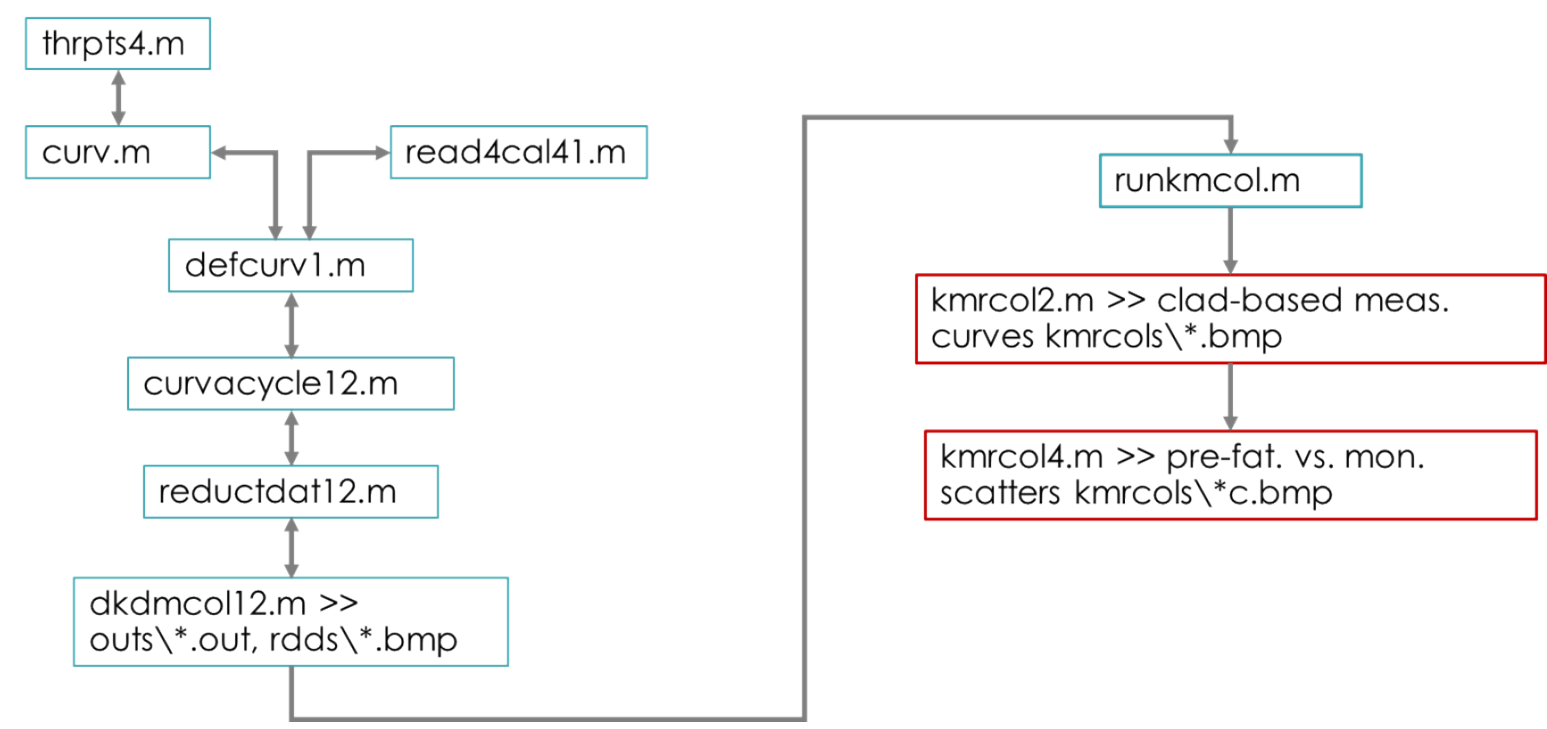

Figure 13. Measurement data processing flowchart.

\subsection{SPECIMEN FATIGUE CURVES}

1. Create subdirectory 'rdds' in $\backslash$ CIRFT.

2. Call readcal41.m for moment calculation.

3. Call curvecycle12 for curvature with sensor spacing correction, rigidity, hysteresis.

4. Call reductdat12 for moment-curvature loops at N.

5. Call dkdmcol12.m for moment-curvature loops at selected interruptions.

\subsection{1 read4cal41.m}

function [t, dsp, load, mmnt, flct1, flct2, flct3, ttltxt] = read4cal41(testnum, reset_renew, iffigure, ifprint);

$\% \quad$ mfile: read4cal41.m

$\% \quad$ read data block, calculate moment, smooth and reset LVDT, and truncate data

$\%$ data flow: readbose $01->$ readrose $12->$ read4cal-> curvacycle-> reductdat1-> dkdmcol

$\%$ example:

$\% \quad$ [t, dsp, load, mmnt, flct1, flct2, flct3, ttltxt] = read4cal41('test00101', 1, 1, 1);

$\% \quad$ toolbox required: Curve Fitting 


\subsection{2 curvacycle12.m}

function [mss_m, kss_k, frg, um, dk, dm, kmin, kmax, mmin, mmax, Lgo2, dh] = curvacycle12(testnum, reset_renew, iffigure, ifprint);

$\% \quad$ mfile: curvacycle $12 . \mathrm{m}$

$\% \quad$ dataflow: readbose $01 \rightarrow$ readbose $12 \rightarrow$ read4cal $\rightarrow$ curvacycle $\rightarrow$ reductdat $1 \rightarrow$ dkdmcol

$\% \quad$ calculate curvature; correct sensor spacing, rigidity, and hysteresis; smooth and truncate data

$\%$ example:

$\% \quad$ close all; clear all; [mss_m,kss_k,frg,um,dk,dm,kmin,kmax,mmin,mmax,Lgo2,dh] = curvacycle12('test00201',1,1,0);

$\% \quad$ functions called: read4cal41.m, defcurv1.m

$\%$ toolbox required: Curve Fitting

Figure 14 shows the quantities in defining flexural rigidity and hysteresis. For those used in defining stiffness and bending hysteresis, rotation is used as a substitute for curvature.

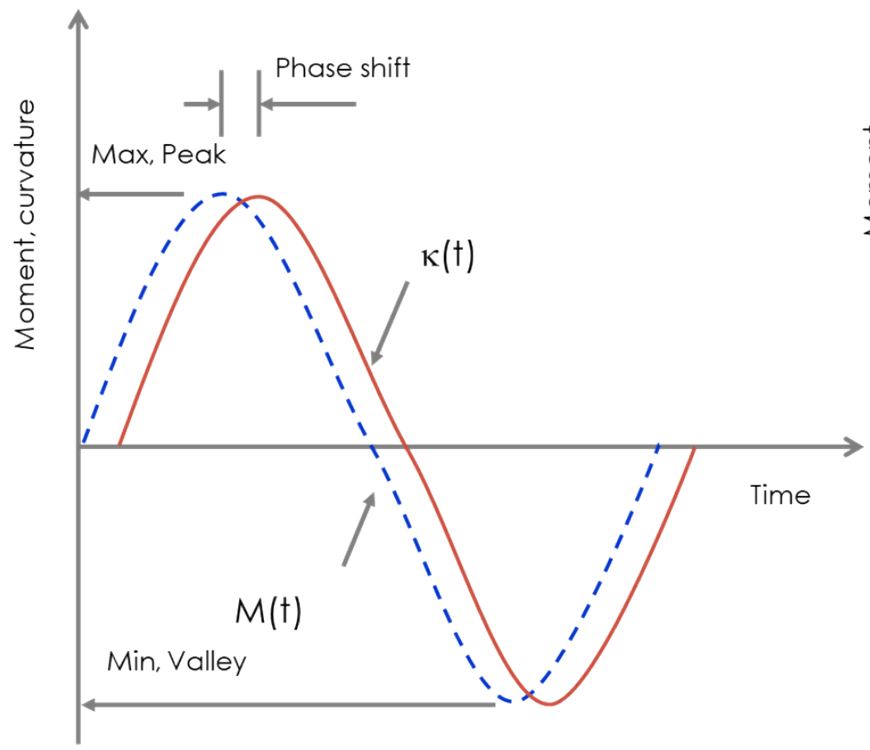

(a)

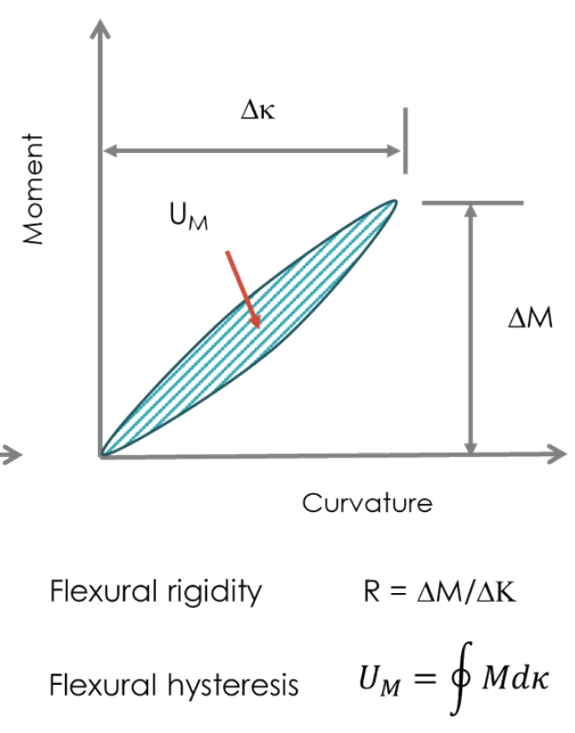

(b)

Figure 14. Quantities are introduced above to characterize the bending mechanical properties of the rod: (a) timed data, (b) moment vs. curvature loop.

\subsection{3 defcurv1.m}

function [ks_opt, ths_opt, Lgo2_opt, dh_opt] = defcurv1(f1s, f2s, f3s, ifcor, iffigure, ifprint);

$\% \quad$ mfile: defcurv1.m

$\% \quad$ functions called: curv.m

Output:

ks_opt—curvature, array

ths_opt, rotation angle, array

Lgo2_opt - half gage length 
dh_opt - sensor spacing correction

\subsection{4 curv.m}

function $[k, t h]=\operatorname{curv}(f 1, f 2, f 3, d h$, iffigure, ifprint $)$;

$\% \quad$ mfile: curv.m

$\%$ get curvature and rotation angle using derivatives of quadratic polynomials

$\% \quad$ example: close all; clear all; $[\mathrm{k}, \mathrm{th}]=\operatorname{curv}(\mathrm{f} 1, \mathrm{f} 2, \mathrm{f} 3, \mathrm{dh}$, iffigure, ifprint $)$;

$\%$ functions called: thrpts4.m

\subsection{5 thrpts4.m}

function $\mathrm{c}=\operatorname{thrpts} 4(\mathrm{~d} 1, \mathrm{~d} 2, \mathrm{~d} 3, \mathrm{~h})$;

$\% \quad$ mfile: thrpts4.m

$\%$ define coefficients of quadratic polynomials

$\% \quad \mathrm{y}=\mathrm{c} 0 * \mathrm{x}^{\wedge} 2+\mathrm{c} 1 * \mathrm{x}+\mathrm{c} 2 ; \mathrm{c}=[\mathrm{c} 0 ; \mathrm{c} 1 ; \mathrm{c} 2]$

\subsection{6 reductdat12.m}

function [frg, um, dk, dm, kmin, kmax, mmin, mmax, Lgo2, dh] = reductdat12(N, samples, reset_renew, mond, ifprint);

$\% \quad$ mfile: reductdat12.m

$\%$ dataflow: readbose01-> readbose12-> read4cal-> curvacycle-> reductdat 1 -> dkdmcol

$\%$ plot moment-curvature loops at cycle $\mathrm{N}$

$\%$ functions called: curvacycle $12 . \mathrm{m}$

\subsection{7 dkdmcol12.m}

function fig $=\mathrm{dkdmcol} 12($ dirnam, meascol, Nset, ifprint $)$;

$\% \quad$ mfile: dkdmcol12.m

$\% \quad$ plot fatigue curves of measurement quantities

$\%$ dataflow: readbose01-> readrose12-> read4cal-> curvacycle-> reductdat1-> dkdmcol

$\% \quad$ example: close all; clear all; fig = dkdmcol12('D1', 'measd1.inp', 3, 1);

$\%$ functions called: reductdat $12 . \mathrm{m}$

Output:

meas*.out

$\mathrm{Nt}$ - cycles accumulated

$\mathrm{fr}(1,:)$ - flexural rigidity at the first measurement amplitude

um( $(1,:)$ - flexural hysteresis

$\operatorname{det} \mathrm{R}(1,:)$ - rigidity phase angle

$\mathrm{dk}(1,:)$ - curvature range

$\operatorname{dm}(1,:)$ - moment range

$\operatorname{kmin}(1,:)$ - minimum curvature

kmean $(1,:)$ - mean curvature 
$\operatorname{kmax}(1,:)$-maximum curvature

$\operatorname{mmin}(1,:)$-minimum moment

mmean $(1,:)$ - mean moment

$\operatorname{mmax}(1,:)$-maximum moment

Lgo2(1,:) - half gage length

$\mathrm{dh}(1,:)$ - sensor spacing correction

\subsection{ASSEMBLED FATIGUE CURVES}

1. Make subdirectory 'kmrcols' in \CIRFT.

2. Call runkmcol.m to generate output of measurement for all the specimens.

3. Call kmrcol2.m to assemble fatigue curves according to cladding type.

4. Call kmrcol4.m to plot measured quantities vs. monitored ones.

\subsection{1 runkmcol.m}

function runkmcol(dynfile)

$\% \quad$ mfile: runkmcol.m

$\% \quad$ batch process the measurement data for all the specimens

$\% \quad$ example: close all; clear all; runkmcol('dynasis.inp')

$\% \quad$ functions called: dkdmcol12.m

\subsection{2 kmrcol2.m}

function kmrcol2(dynfile, clad, ifprint)

$\% \quad$ mfile: kmrcol2.m

$\% \quad$ assemble fatigue curves for all the specimens/the first amplitude

$\% \quad$ dataflow: runkmcol $\rightarrow \mathrm{kmrcol} 2$

$\% \quad$ example: close all; clear all; kmrcol2('dynasis5.inp','M5',1);

For input file, ${ }^{*}$.inp, please refer to Section 7.3

\subsection{3 kmrcol4.m}

function kmrcol4(dynfile)

$\% \quad$ mfile: kmrcol4.m

$\% \quad$ compile dynafile ->runkmcol -> kmrcol4

$\% \quad$ correlate measurement to monitoring

$\% \quad$ example: close all; clear all; kmrcol4('dynasis5.inp'); 


\section{DATA PROCESSING FOR MONITORING}

Data processing for monitoring works at the specimen level and group level. Data for individual specimen are processed in each data block and then re-integrated to the session/period. For groups of specimens, monitoring quantities and properties will be extracted and/or assembled in terms of fatigue curves and scatter plots.

Figure 15 provides an overview of functions and the relationships among them. The comment lines of each function and supporting sub-functions are provided in the following subsections.
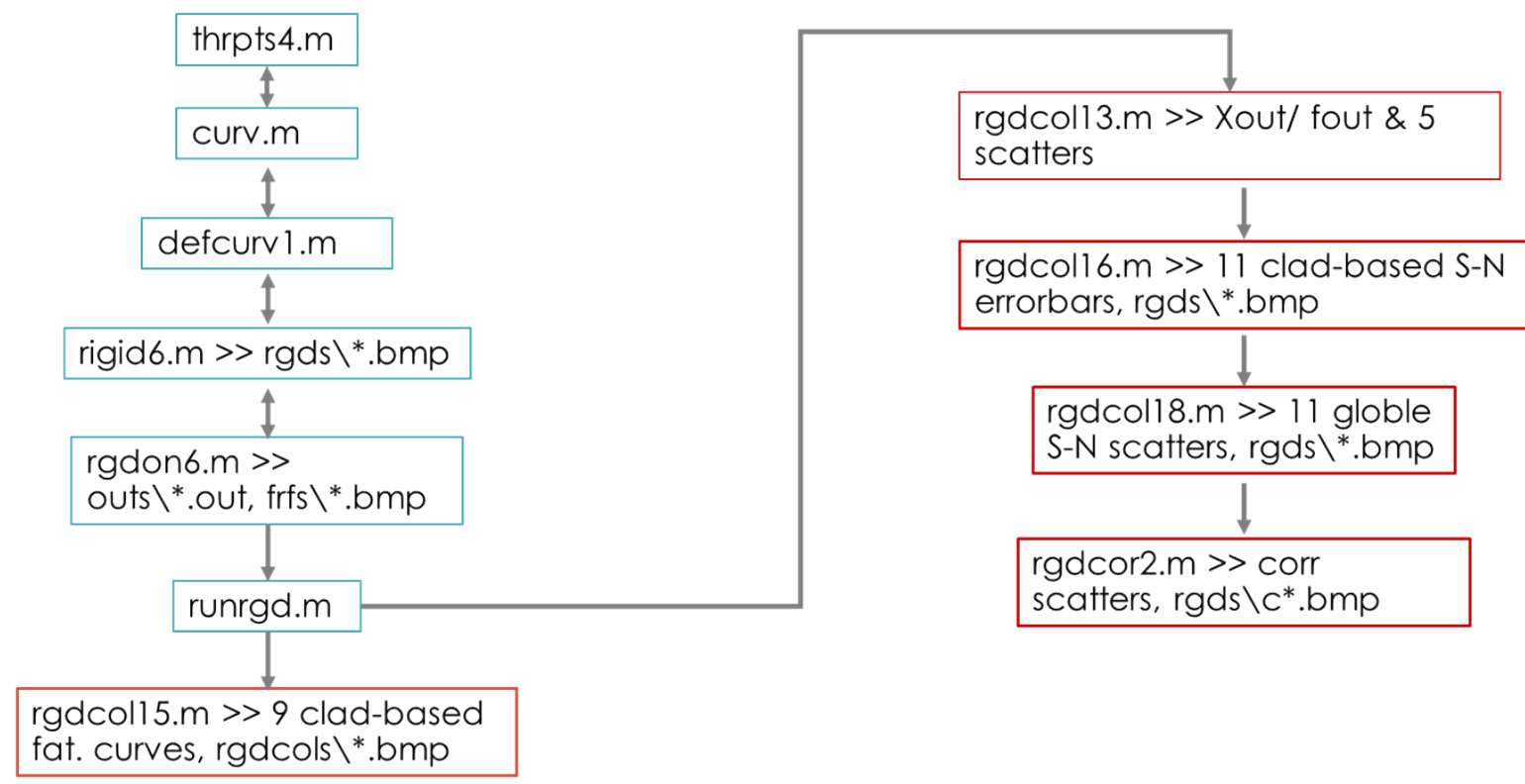

Figure 15. Flowchart of monitoring data processing and functions.

\subsection{FATIGUE DATA AND PLOTS FOR INDIVIDUAL DATA BLOCK}

1. Create data directory for data and plot output: \ldcs.

2. Call rigid6 to get moment and curvature plots in time and frequency domains for individual test block.

\subsection{1 rigid6.m}

function [t0, pk, stf, Us, sphs1, rg, Um, rphs1, Lgo2, dh] = rigid6(ddr, testnum, iffigure, ifprint);

$\% \quad$ mfile: rigid6.m

$\% \quad$ read and process data block

$\%$ example:

$\% \quad$ close all; clear all; [t0,pk, stf,Us,sphs1, rg,Um,rphs1, Lgo2,dh] = rigid6('MOX07','test00310', $1,0)$;

$\% \quad$ functions called: defcurv1.m, curv.m, thrpts4.m, xfilter.m

Output:

t0 - starting time of block 
$\mathrm{pk}$ — data structure for peaks/valleys: moment $\mathrm{mt}$, motor 1 displacement $\mathrm{d} 1$, motor 2 displacement $\mathrm{d} 2$, deflection at LVDT2 f2, curvature kp, rotation angle th, rigid-arm loading-point displacements $\mathrm{u} 1, \mathrm{u} 2$ stf-bending stiffness

Us_-bending hysteresis

sphs1-stiffness phase angle

rg-flexural rigidity

Um-flexural hysteresis

rphs 1 - rigidity phase angle

Lgo2 - half gage length

dh-sensor spacing correction

\subsection{2 xfilter.m}

function [ts, xf, ffs, xfs, xmag, xphs] = xfilter(ts, ps, ttltext, iffigure);

$\% \quad$ mfile: xfilter.m

$\% \quad$ de-noise using Gaussian filter at dc and fundamental frequency

\subsection{SPECIMEN FATIGUE CURVES}

1. Create data directory for data and plot output: \outs, \frfs.

2. Call rdgon6 to obtain fatigue curves for specimen.

\subsection{1 rgdon6.m}

function rgdon6(ddr, moncol, ifkoff, iffigure, ifprint);

$\% \quad$ mfile: rgdon6.m

$\%$ plot on-line monitoring data

$\% \quad$ example: close all; clear all; rgdon6('MOX07','monmox07.inp',0,0,0);

$\% \quad$ mfiles called: rigid6.m

The arguments include data directory name, ddr; file name for monitoring summary input, moncol; control for curvature offset (koffset) adjustment, ifkoff; additional controls on figure and print. Figure 16 illustrates how the adjustment is applied to koffset. This may be needed if the cyclic test is interrupted by events like measurements, or unplanned events like power outage. In all these events, the curvature offset status of the rod is expected to remain the same as that of the last data scan and is adjusted as necessary.

Output:

mon*.out consists of the following columns

Ns-number of cycles

Mts - peak and valley of moment

Kps - peak and valley of curvature

$\mathrm{f} 2 \mathrm{~s}$ - peak and valley of deflection

d1s - peak and valley of displacement at motor 1

$\mathrm{d} 2 \mathrm{~s}$ - peak and valley of displacement at motor 2

stfs - bending stiffness

Uss-bending hysteresis

sph $1 \mathrm{~s}$ - stiffness phase angle

rgs-flexural rigidity 
Ums - flexural hysteresis

rph1s - rigidity phase angle

Lgo2s - half gage length

dhs-sensor spacing correction

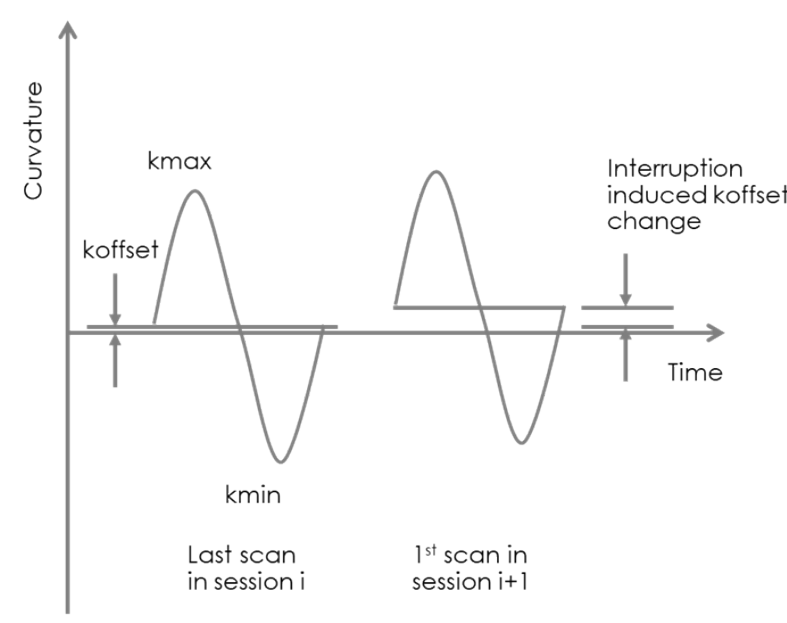

(a)

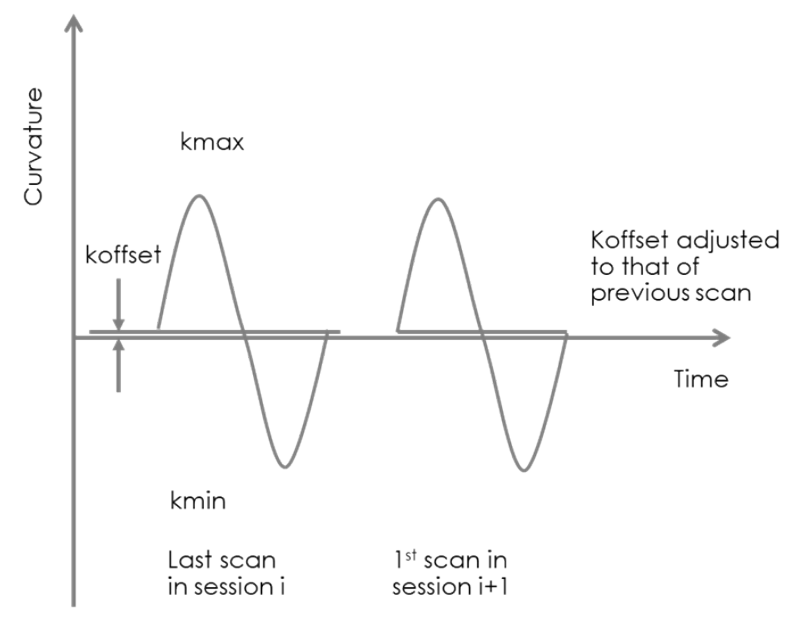

(b)

Figure 16. Diagram showing koffset adjustment related to the measurement interruption or equivalent: (a) before and (b) after adjustment.

\subsection{ASSEMBLED FATIGUE CURVES}

1. Prepare summary input. dyna*.inp, dyna*.stt.

2. Make data directory $\backslash \mathrm{rgds}$, $\backslash$ rgdcols for scatters and fatigue curves, respectively.

3. Call runrgd.m to batch process test data of all the specimens, if not processed yet.

4. Call rgdcol15.m to assemble fatigue curves according to cladding type.

\subsection{1 runrgd.m}

function runrgd(dynfile)

$\% \quad$ mfile: runrgd.m

$\%$ date: $9-16-2014$

$\% \quad$ example: close all; clear all; runrgd('dynasis.inp')

\subsection{2 rgdcol15.m}

function rgdcol15(dynfile, clad, ifprint)

$\% \quad$ mfile: rgdcoll5.m

$\%$ dataflow: runrgd -> rgdcol15

$\% \quad$ plot fatigue curves based on cladding type

$\%$ example:

$\% \quad$ close all; clear all; rgdcol15('dynasis5.inp', 'M5', 1); 
Input:

dyna*.inp

\begin{tabular}{|c|c|c|c|c|c|c|c|c|c|}
\hline D2 & D2 & $30 \mathrm{AD} 05-2050-2203$ & 8.34 & 9.43 & 8.34 & 0 & 60 & 6.10 & $1.33 \mathrm{E}+05$ \\
\hline & 1 & 4 & & & & & & & \\
\hline D1 & D1 & $30 \mathrm{AD} 05-2630-2783$ & 8.34 & 9.43 & 8.34 & 0 & 120 & 12.19 & $2.23 \mathrm{E}+04$ \\
\hline & 1 & 0 & & & & & & & \\
\hline $\mathrm{S} 2$ & S2 & 30AE14-672-825 & 8.34 & 9.46 & 8.34 & 0 & 175 & 17.78 & $1.63 \mathrm{E}+03$ \\
\hline & 1 & 0 & & & & & & & \\
\hline D3 & D3 & 30AE14-2850-3003 & 8.34 & 9.46 & 8.34 & 0 & 120 & 12.19 & $9.80 \mathrm{E}+03$ \\
\hline & 1 & 0 & & & & & & & \\
\hline D4 & D4 & 30AE14-3156-3309 & 8.34 & 9.46 & 8.34 & 0 & 60 & 6.10 & $1.13 \mathrm{E}+05$ \\
\hline & 1 & 0 & & & & & & & \\
\hline S3 & S3 & 3A1F05-1853-2006 & 8.42 & 9.46 & 8.42 & 0 & 175 & 17.78 & $1.30 \mathrm{E}+03$ \\
\hline & 1 & 0 & & & & & & & \\
\hline D12 & D12 & 3A1F05-2025-2178 & 8.42 & 9.46 & 8.42 & 0 & 100 & 10.16 & $4.82 \mathrm{E}+04$ \\
\hline & 1 & 0 & & & & & & & \\
\hline D9 & D9 & 3A1F05-3214-3367 & 8.42 & 9.46 & 8.42 & 0 & 100 & 10.16 & $3.45 \mathrm{E}+03$ \\
\hline & 1 & 2 & & & & & & & \\
\hline D14 & D14 & $3 \mathrm{~A} 1 \mathrm{~F} 05-3367-3520$ & 8.42 & 9.46 & 8.42 & 0 & 50 & 5.08 & $2.14 \mathrm{E}+05$ \\
\hline & 1 & 0 & & & & & & & \\
\hline D8 & D8 & 3D8E14-1178-1331 & 8.38 & 9.49 & 8.38 & 0 & 60 & 6.10 & $2.12 \mathrm{E}+05$ \\
\hline & 1 & 0 & & & & & & & \\
\hline 07 & D7 & 3D8E14-2412-2565 & 8.38 & 9.49 & 8.38 & 0 & 60 & 6.10 & $1.91 \mathrm{E}+05$ \\
\hline
\end{tabular}

dyna*.stt

$\begin{array}{llllllllll}\text { D2 } & \text { 30AD05 } & 2050 & 10.26 & 543 & 52 & \text { zone1 } & \text { M5 } & \text { AR } & 0 \\ \text { D1 } & \text { 30AD05 } & 2630 & 10.26 & 543 & 52 & \text { zone1 } & \text { M5 } & \text { AR } & 0 \\ \text { S2 } & \text { 30AE14 } & 672 & 10.28 & 560 & 52 & \text { zone1 } & \text { M5 } & \text { HS } & 1 \\ \text { D3 } & \text { 30AE14 } & 2850 & 10.28 & 560 & 52 & \text { zone1 } & \text { M5 } & \text { HT } & 0 \\ \text { D4 } & \text { 30AE14 } & 3156 & 10.28 & 560 & 52 & \text { zone1 } & \text { M5 } & \text { HT } & 0 \\ \text { S3 } & \text { 3A1F05 } & 1853 & 9.92 & 520 & 50 & \text { zone1 } & \text { LT } & \text { ST } & 1 \\ \text { D12 } & \text { 3A1F05 } & 2025 & 9.92 & 520 & 50 & \text { zone1 } & \text { LT } & \text { AR } & 0 \\ \text { D9 } & \text { 3A1F05 } & 3214 & 9.92 & 520 & 50 & \text { grid6 } & \text { LT } & \text { AR } & 0 \\ \text { D14 } & \text { 3A1F05 } & 3367 & 9.92 & 520 & 50 & \text { zone2 } & \text { LT } & \text { AR } & 0 \\ \text { D8 } & \text { 3D8E14 } & 1178 & 10.05 & 555 & 55 & \text { grid2 } & \text { ZL } & \text { AR } & 0 \\ \text { D7 } & \text { 3D8E14 } & 2412 & 10.05 & 555 & 55 & \text { zone1 } & \text { ZL } & \text { AR } & 0\end{array}$

\subsection{FATIGUE SCATTER PLOTS}

1. Make data directory $\backslash$ rgds, for fatigue scatter plots.

2. Prep dyna*.daq.

3. Call rgdcol13.m to calculate mean/std of fatigue quantities for error bars.

4. Call rgdcol16.m to obtain error bars of monitored quantities according to cladding type.

5. Call rgdcol18.m to obtain scatter plots of monitored quantities according to fuel type.

6. Call rgdcor2.m for monitored quantities correlation. 


\subsection{1 $\quad$ rgdcol13.m}

function $[$ Xout, fig $]=$ rgdcol13(dynafile);

$\% \quad$ mfile: rgdcoll3.m

$\% \quad$ integrate mean/ std of original and equivalent quantities of specimens in the group

$\%$ example:

$\% \quad$ close all; clear all; [Xout, fig] $=$ rgdcol13('dynasis5.inp');

Output:

dyna*1.out

tnn-specimen number

idc-ID of cladding

odc-OD of cladding

diap_diameter of pellet

fa-load amplitude

$\mathrm{nf}$ - number of cycles to failure

fail-status of failure

mta - mean of moment amplitude

mta_std - standard deviation of moment amplitude

$\mathrm{kpa}-$ mean of curvature amplitude

kpa_std — standard deviation of curvature amplitude

$\mathrm{kpm}$ - mean of extreme curvature

$\mathrm{kpm} \_$std — standard deviation of extreme curvature

dyna*2.out

tnn-specimen number

eis - mean of rigidity

eis_std—standard deviation of rigidity

Ums - mean of rigidity hysteresis

UMs_std — standard deviation of rigidity hysteresis

rph $1 \mathrm{~s}$ - mean of rigidity phase angle

rph1s_std — standard deviation of rigidity phase angle

sga - mean of equivalent stress amplitude

sga_std — standard deviation of equivalent strain amplitude

epa-mean of extreme equivalent strain amplitude

epa_std — standard deviation of equivalent strain amplitude

epm- mean of extreme equivalent strain

epm_std — standard deviation of extreme equivalent strain

Lg2- mean of half gage length

Lg2_std - standard deviation of half gage length

$\mathrm{dth}-$ mean of sensor spacing correction

dth_std—standard deviation of sensor spacing correction

\subsection{2 rgdcol16.m}

function fig = rgdcol16(dynfile, clad $)$; 
$\% \quad$ mfile: rgdcoll6.m

$\% \quad$ runrgd $\rightarrow \operatorname{rgdcol} 16$

$\%$ example:

$\% \quad$ close all; clear all; fig= rgdcol16('dynasis5.stt','M5');

\subsection{3 rgdcol18.m}

function fig $=$ rgdcol18(dynafile);

$\% \quad$ mfile: rgdcol18.m

$\% \quad$ runrgd $\rightarrow \operatorname{rgdcol} 18$

$\%$ example:

$\% \quad$ close all; clear all; fig= rgdcol18('dynasis5.stt');

Additional input:

dyna*.daq is shown below as an example, where flag $=0$, no issue; flag $=1$, disp/defl in question; flag $=2$ load in question

$\begin{array}{ll}\text { D2 } & 0 \\ \text { D1 } & 0 \\ \text { S2 } & 0 \\ \text { D3 } & 0 \\ \text { D4 } & 0 \\ \text { S3 } & 0 \\ \text { D12 } & 0 \\ \text { D9 } & 0 \\ \text { D14 } & 0 \\ \text { D8 } & 0 \\ \text { D7 } & 1 \\ \ldots & \end{array}$

\subsection{4 rgdcor $2 . m$}

function fig $=\operatorname{rgdcor} 2($ dynafile $)$;

$\% \quad$ mfile: rgdcor $2 . m$

$\% \quad$ runrgd $\rightarrow$ rgdcor

$\%$ example:

$\% \quad$ close all; clear all; fig= rgdcor2('dynasis5.stt'); 


\section{POTENTIAL APPLICATIONS}

In the United States, both the dry storage and total inventories of SNF increased rapidly beginning in the year 2000. The Electric Power Research Institute projected that there would be 32,000 MTU of SNF in dry storage by 2020 stored in approximately 2,900 dry storage cask systems. Total SNF discharges by 2020 were projected to be approximately 86,000 MTU. By 2060, at which time all of the currently operating nuclear power plants will have reached the end of their renewed operating licenses, there would be approximately $136,600 \mathrm{MTU}$ of SNF in storage at reactor sites. ${ }^{50}$ The structural integrity of SNF after dry storage and during transportation to disposition sites needs to be determined to facilitate all the options for disposition and to maintain retrievability and normal back-end fuel cycle operations.

Meanwhile, in the United States, the discharge BU of SNF was seen to be crossing a threshold level and turning into high-BU for pressure water reactors at around 2001 and boiling water reactors at around 2015. The structure of nuclear fuels was altered substantially after in-reactor service and subsequent storage. Evaluation and study must therefore address the changes from the mentioned processes.

The CIRFT technology can address the technical challenges and issues. Thus, the data processing package developed is anticipated to have a very promising market.

The CIRFT hardware and related software package can be used in applications for which bending testing and characterization of materials and structures are needed, such as rods and beams. 


\section{CONCLUSION}

A data processing software package has been introduced. The package was developed using MATLAB with the aid of the Curve Fitting Toolbox to analyze and process data associated with the evaluation of spent nuclear fuel rods using the ORNL-developed cyclic integrated reversible bending fatigue testing (CIRFT). The package consists of four modules: pre-processing, data processing for static testing, data processing for monitoring, and data processing for measurements. CIRFT data are structured with multiple levels associated with group, specimen, session, and scan/ block. The degree of complexity of the data structure depends on whether a test is static or cyclic.

The test results are presented in figures, scatter plots, and tables. For static testing, the output in tables provides bending mechanical properties and characteristic points of moment-curvature relation: flexural rigidities in linear segments of loading and unloading stages, intersection points between characteristic segments of the curve, and equivalent stress and strain quantities. For cyclic testing, the table output lists control and fatigue life and responsive/dependent quantities including moment, curvature, flexural rigidity, flexural hysteresis, rigidity phase angle, and equivalent stresses and strains. In addition, derivatives such as half-gage length and sensor spacing correction are included. The output also provides standard deviations of the reported quantities when they are applicable or available.

The data processing package can serve as a fundamental characterization tool in mechanical study of materials. The package is intended primarily for data processing for the CIRFT process and can also be used in applications for which similar testing requirements exist. The package is a critical element for CIRFT to be developed as an important nuclear testing facility and for many users in the field of storage, handling, and transportation of used fuels.

The CIRFT adopts a very unique approach to characterizing the mechanical behavior of rod-shaped specimens by using curvature as a measurement of deformation. The data processing package introduced in this report addresses the relevant technical issues with such measurement, including sensor spacing correction, frictional resistance, and signal denoising. There are several aspects that need to be developed to have the package more versatile and more friendly.

For example, the current data analysis focuses on the curvature. The curvature is defined over the whole gage section of specimen and limited with the assumption that the continuity of specimen is largely maintained. The curvature may not be sensitive to the deformation if the damage takes place outside of sensor range. As a result of that, there is a need to investigate the deflection data to understand the effect of the damage on the curvature measurement. Also, part of the CIRFT data can be displayed online, but the data processing as described in this report is mostly conducted offline. There is a need to interface the package with commercial software in future to present the bending and mechanical performance data in real time. 


\section{ACKNOWLEDGMENTS}

The work in this report was jointly sponsored by the Office of Nuclear Regulatory Research, the US Nuclear Regulatory Commission (NRC) and the US Department of Energy (DOE) Used Fuel Disposition Campaign programs under DOE contract DE-AC05-00OR22725 with UT-Battelle, LLC.

The authors would like to thank NRC program managers Michelle Bales and Patrick Raynaud, ORNL program managers Bruce Bevard and Rob Howard, and Pacific Northwest National Laboratory program manager Harold Adkins for providing guidance and support. The authors are grateful to Drs. Edgar LaraCurzio, Weiju Ren, and Lianshan Lin of ORNL for their time to review this manuscript and their comments. 


\section{REFERENCES}

${ }^{1}$ NRC, General Standards for All Packages, §43(f) in Title 10, Code of Federal Regulations, Part 71, Packaging and Transportation for Radioactive Material, US Nuclear Regulatory Commission, Washington, DC, Dec. 6, 2010.

${ }^{2}$ H. C. Chu, S. K. Wu, R. C. Kuo, Hydride reorientation in Zircaloy-4 cladding, J. Nucl. Mater., 373, 2008, 319-327.

${ }^{3}$ W. Weibull, Fatigue and Analysis of Results, Pergamon Press, New York, 1961, pp. 31-65.

${ }^{4}$ A. E. Carden, Fatigue at elevated temperatures: a review of test methods, in Fatigue at Elevated Temperatures, ASTM STP 520, Eds. A. E. Carden, A. J. McEvily, and C. H. Wells, ASTM, Philadelphia, 1973, pp. 195-223.

${ }^{5}$ ASTM, Manual on Low Cycle Fatigue Testing, ASTM STP 465, ASTM, Philadelphia, 1969, pp. 149162.

${ }^{6}$ ASTM E-9 Committee on Fatigue, Handbook of Fatigue Testing, ASMT STP 566, Ed. S. R. Swanson, ASTM, Philadelphia, 1974, pp. 19-84.

${ }^{7}$ ASTM B 593-96, Standard Test Method for Bending Fatigue Testing for Copper-Alloy Spring Materials, ASTM International, West Conshohocken, PA.

${ }^{8}$ M. A. Abojaradeh, M. W. Witczak, M. S. Mamlouk, and K. E. Kaloush, Validation of initial and fatigue stiffness definition in flexure fatigue test for hot mix asphalt, J. Test. Eval., 35, 2007, 1-8.

${ }^{9}$ T. K. O’Brien, A. D. Chawan, R. Krueger, and I. L. Paris, Transverse tension fatigue life characterization through flexure testing of composite materials, Int. J. Fatigue, 24, 2002, 127-145.

${ }^{10} \mathrm{~W}$. D. Bowman, End constraint and alignment effects in three and four point tension-compression bending tests, in Factors That Affect the Precision of Mechanical Tests, ASTM STP 1025, Eds. R. Papirno and H. C. Weiss, ASTM, Philadelphia, 1989, pp. 174-184.

${ }^{11}$ L. Bertele, A. Papack, and K.-H. Wichmann, Testing Device for Performing Four Point Fatigue Strength Tests under Alternating Bending Stresses, US Patent 5231882, Aug. 3, 1993.

${ }^{12}$ T. B. Zineb, A. Sedrakian, and J. L. Billoet, An original pure bending device with large displacements and rotations for static and fatigue tests of composite structures, Composites Part B: Engineering, 34, 2003, 447-458.

${ }^{13}$ T. Zhai, Y. G. Xu, J. W. Martin, A. J. Wilkinson, and G.A.D. Briggs, A self-aligning four-point bend testing rig and sample geometry effect in four-point bend fatigue, Int. J. Fatigue, 21, 1999, 889-894.

${ }^{14}$ M. M. Gharnian, S. Krishnasamy and A. N. Sherbourne, Inelastic deformations of mild-steel beams under symmetrical and unsymmetrical cyclic bending, Experimental Mechanics, Feb 1974, 49-56.

${ }^{15}$ R. Lagneborg and R. Attermo, The effect of combined low-cycle fatigue and creep on the life of austenitic stainless steel, Metall. Trans., 2, 1971, 1821-1827.

${ }^{16}$ N. Gao, M. W. Brown, K. J. Miller, and P. A. S. Reed, An effective method to investigating short crack growth behavior by reverse bending testing, Int. J. Fatigue, 29, 2007, 565-574.

${ }^{17}$ P. G. Forrest and A. B. Penfold, New approach to thermal fatigue testing, Engineering (London), 192, $1961,522-523$.

${ }^{18}$ D. J. White, The effect of environment and hold time on the high strain fatigue endurance of $0.5 \%$ Mo steel, Proc. Inst. Mech. Eng., 184, 1969, 223-240. 
${ }^{19}$ A. D. Batte, R. A. T. Dawson, E. B. Evans, and G. Thomas, Reverse-bend testing to obtain long-term endurance data, in Techniques for High Temperature Fatigue Testing, Eds. G. Sumner and V. B. Livesey, Elsevier Appl. Sci. Pub., London, 1985, pp. 97-115.

${ }^{20}$ A. A. Tayebali, J. A. Deacon, and C. L. Monismith, Development of dynamic flexural beam fatigue test system, National Research Council, Transportation Research Board, Transportation Research Record, 1545, 1996, 89-97.

${ }^{21}$ J.-A. J. Wang, H. Wang, Y. Yan, R. Howard, and B. Bevard, High Burn-up Spent Fuel Vibration Integrity Study Progress Letter Report (Out-of-cell Fatigue Testing Development-Task 2.1), ORNL/ TM-2010/288, Jan 2011.

22 J.-A. J. Wang, H. Wang, T. Tan, H. Jiang, T. Cox, Y. Yan, Progress Letter Report on U-frame Test Setup and Bending Fatigue Test for Vibration Integrity Study (Out-of-cell Fatigue Testing Development-Task 2.2), ORNL/TM- 2011/531, January 2012.

${ }^{23}$ J.-A. J. Wang, H. Wang, T. Cox, Y. Yan, Progress Letter Report on U-Frame Test Setup and Bending Fatigue Test for Vibration Integrity Study (Out-of-cell Fatigue Testing Development-Task 2.3), ORNL/TM- 2012/417, August 2012.

${ }^{24}$ J.-A. J. Wang, H. Wang, T. Cox, C. Baldwin, Progress Letter Report on Bending Fatigue Test System Development for Spent Nuclear Fuel Vibration Integrity Study (Out-of-cell Fatigue Testing Development—Task 2.4), ORNL/TM- 2013/225, July 2013. www.osti.gov/servlets/purl/1091652/.

${ }^{25}$ H. Wang, J.-A. J. Wang, T. Tan, H. Jiang, T. S. Cox, R. L. Howard, B. B. Bevard, and M. E. Flanagan, Development of U-frame bending system for studying the vibration integrity of spent nuclear fuel, $J$. Nucl. Mater., 440, 2013, 201-213.

${ }^{26}$ J.-A. J. Wang and H. Wang, B. Bevard, The Development of Reversible Bending Fatigue Tester and Its Application to High Burn-Up Spent Nuclear Fuel Integrity Study under Normal Transportation Vibration, ORNL/TM- 2013/573, August 2014.

27 J.-A. J. Wang, H. Wang, and T. Tan, Reversal Bending Fatigue Testing, US Patent, US 8,863,585 B2, Oct. 21, 2014.

${ }^{28}$ J.-A. J. Wang, H. Wang, B. B. Bevard, R. L. Howard, and M. E. Flanagan, Reversible bending fatigue test system for investigating vibration integrity of spent nuclear fuel during transportation, Packaging, Transport, Storage \& Security of Radioactive Material, 25, 2014, 119-132.

${ }^{29}$ H. Jiang, J. J.-A. Wang, H. Wang, Reversible bending fatigue testing system design concepts for spent fuel vibration integrity study, Proc. ASME 2014 Pressure Vessels \& Piping Conference, Anaheim, California, July 20-24, 2014. Paper No. PVP2014-29117.

${ }^{30}$ J.-A. J. Wang, H. Wang, Progress Letter Report on Reversal Bending Fatigue Testing of Zry-4 Surrogate Rod (Out-of-cell Fatigue Testing Development-Task 2.4), ORNL/TM- 2013/297, August 2013.

${ }^{31} \mathrm{H}$. Wang, J.-A. J. Wang, Bending testing and characterization of surrogate nuclear fuel rods made of Zircaloy-4 cladding and aluminum oxide pellets, J. Nucl. Mater., 479, 2016, 470-482.

${ }^{32}$ H. Wang, J.-A. J. Wang, Experimental Study on Surrogate Nuclear Fuel Rods Under Reversed Cyclic Bending, in Fatigue and Fracture Test Planning, Test Data Acquisitions, and Analysis, ASTM STP 1598, Z. Wei, K. Nikbin, P. McKeighan, and G. Harlow, Eds., ASTM International, West Conshohocken, PA, 2017, pp. 19-36. 
${ }^{33}$ J.-A. Wang and H. Wang, B. B. Bevard, R. Howard, 2014 Semi-annual Progress Letter Report on Used Nuclear Fuel Integrity Study in Transportation Environments, prepared for US Department of Energy, ORNL/TM-2014/63, April 2014.

${ }^{34}$ J.-A. Wang, H. Wang, Mechanical Fatigue Testing of High-burnup Fuel for Transportation Applications, NUREG/CR-7198/ ORNL/TM-2014/214, prepared for Office of Nuclear Regulatory Research, US Nuclear Regulatory Commission, May 2015.

35 J.-A. J. Wang, H. Wang, and H. Jiang, FY14 status report: CIRFT Testing Results on High Burnup UNF, ORNL/TM-2014/310, September 2014.

${ }^{36}$ J.-A. J. Wang, H. Wang, and H. Jiang, FY15 Status Report: CIRFT Testing of Used Nuclear Fuel Rods from the Limerick Generating Station Boiling Water Reactor, ORNL/TM-2015/171, April 30, 2015.

${ }^{37}$ J.-A. J. Wang, H. Wang, H. Jiang, and Y. Yan, CIRFT Testing of High-burnup Used Nuclear Fuel Rods from Pressurized Water Reactor and Boiling Water Reactor Environments, ORNL/TM-2015/313, September 5, 2015.

38 J.-A. J. Wang, H. Wang, FY 2016 Status Report: CIRFT Testing Data Analyses and Updated Curvature Measurements, ORNL/SPR-2016/276, July 24, 2016.

${ }^{39}$ J.-A. Wang, H. Wang, H. Jiang, Y. Yan, FY 2016 Status Report: Documentation of All CIRFT Data including Hydride Reorientation Tests, ORNL/SR-2016/424, September 14, 2016.

${ }^{40}$ J.-A. Wang, H. Wang, ORNL Interim Progress Report on Static CIRFT Testing Curvature Data Update, ORNL/TM-2016/619, Oak Ridge National Laboratory, Oak Ridge, TN, Oct. 20, 2016.

${ }^{41}$ J.-A. Wang, H. Wang, Y. Yan, ORNL Interim Progress Report on Hydride Reorientation CIRFT Tests, ORNL/TM-2016/660, Oak Ridge National Laboratory, Oak Ridge, TN, Oct. 28, 2016.

42 J.-A. Wang, H. Wang, B. B. Bevard, J. M. Scaglione, FY 2017 Status Report: CIRFT Data Update and Data Analyses for Spent Nuclear Fuel Vibration Reliability Study, ORNL/TM-2017/291, Oak Ridge National Laboratory, Oak Ridge, TN, Aug. 8, 2017.

${ }^{43}$ J.-A. Wang, H. Wang, Mechanical fatigue testing of high-burnup fuel for transportation applications, NUREG/CR-7198, Rev. 1/ ORNL/TM-2016/689, prepared for Office of Nuclear Regulatory Research, U.S. Nuclear Regulatory Commission, Oct. 2017.

${ }^{44}$ H. Wang, J.-A. J. Wang, and H. Jiang, Fatigue behavior of spent nuclear fuel rods in simulated transportation environment, Proc. ASME 2017 Pressure Vessels \& Piping Conference, PVP2017, Waikoloa, Hawaii, July 16-20, 2017, Paper Number: PVP2017-65842.

${ }^{45}$ J.-A. Wang, H. Wang, H. Jiang, B. Bevard, High burn-up spent nuclear fuel transport reliability investigation, Nucl. Eng. and Design, 330, 2018, 497-515.

${ }^{46}$ H. Wang, J.-A. J. Wang, R. Montgomery, B. B. Bevard, CIRFT Data Analysis for Sister Rods, ORNL/TM-2019/1451, Oak Ridge National Laboratory, Oak Ridge, TN, Oct. 2020.

${ }^{47}$ R. Montgomery, J.-A. J. Wang, H. Wang, B. B. Bevard, D. Skitt, and O. Martinez, Sister Rod Destructive Examinations (FY20), Appendix F: Cyclic Integrated Reversible-Bending Fatigue Tests, ORNL/SPR-2020/1780, Oak Ridge National Laboratory, Oak Ridge, TN, Nov. 2020.

${ }^{48}$ ElectroForce, LM2 TestBench Test Instrument Reference Manual, 373489-0010, Bose Corp, Eden Prairie, MN 55344, 2013.

${ }^{49}$ ElectroForce, WinTest7 Software Reference Manual, 373490-0010, Bose Corp, Eden Prairie, MN 55344, 2013. 
${ }^{50}$ EPRI, Impacts Associated with Transfer of Spent Nuclear Fuel from Spent Fuel Storage Pools to Dry Storage after Five Years of Cooling, Rev. 1, TR- 1025206, Electric Power Research Institute, Palo Alto, California, August 2012. 